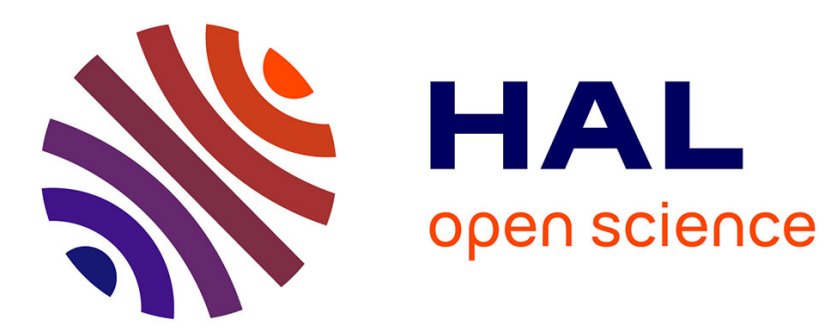

\title{
Subdifferentiation of integral functionals
}

\author{
Emmanuel Giner, Jean-Paul Penot
}

\section{To cite this version:}

Emmanuel Giner, Jean-Paul Penot. Subdifferentiation of integral functionals. 2017. hal-01450022

\section{HAL Id: hal-01450022 \\ https://hal.sorbonne-universite.fr/hal-01450022}

Preprint submitted on 30 Jan 2017

HAL is a multi-disciplinary open access archive for the deposit and dissemination of scientific research documents, whether they are published or not. The documents may come from teaching and research institutions in France or abroad, or from public or private research centers.
L'archive ouverte pluridisciplinaire HAL, est destinée au dépôt et à la diffusion de documents scientifiques de niveau recherche, publiés ou non, émanant des établissements d'enseignement et de recherche français ou étrangers, des laboratoires publics ou privés. 


\section{Subdifferentiation of integral functionals}

\section{Emmanuel Giner $(*)$ and Jean-Paul Penot $(\dagger)^{1}$}

Dedicated to Professor R.T. Rockafellar on the occasion of his eightieth birthday.

Abstract. We examine how the subdifferentials of nonconvex integral functionals can be deduced from the subdifferentials of the corresponding integrand or at least be estimated with the help of them. In fact, assuming some regularity properties of the integrands, we obtain exact expressions for the subdifferentials of the integral functionals. We draw some consequences in terms of duality for such integral functionals, extending in this way the early work of R.T. Rockafellar to the nonconvex case.

Key words. integral functional, integrand, Legendre function, regularity, subdifferential.

Mathematics Subject Classification. 28C99, 49J52, 58Cxx, 46G05

\section{Introduction}

The word "regularity" has many different meanings in mathematics. In nonsmooth analysis the concepts already used by F.H. Clarke [8] (under the term "regularity" we change here into C-D regularity for the sake of clarity), R.T. Rockafellar [52] (under the terms of "geometric derivability", "epidifferentiability", "protodifferentiability" in [52] and elsewhere) and J.-P. Penot (under the term of "softness" [41]) can be completed by a number of different notions. The purposes of such concepts are twofold. First, for the functions or the classes of functions that satisfy such regularity, the number of available subdifferentials is reduced, giving more strength to the notion of subdifferential. Second, regularity enables to get new properties, in particular equalities instead of inclusions (see [8] for instance).

Given two subdifferentials $\partial_{A}$ and $\partial_{B}$, we suggest to say that a function $f$ is $A$-B-regular at some point $x$ of its domain if $\partial_{A} f(x)=\partial_{B} f(x)$. Of course, if $f$ is convex or approximately convex, or of class $C^{1}, f$ is A-B-regular for all usual subdifferentials $\partial_{A}$ and $\partial_{B}$. Since the calculus rules for $\partial_{A}$ and $\partial_{B}$ may be different, in some cases of interest A-B-regularity can be transferred to new functions build from A-B regular functions (see [8] for the important case of C-D regularity or Clarke regularity).

In this survey we focus the attention on the application of such ideas to integral functionals, an important class of functions. Such functions are typically defined on an infinite dimensional space, a $L_{p}$ space, on the contrary of functions defined as parameterized integrals such as

$$
E_{f}(x):=\int_{S} f(s, x) d \mu(s) \quad x \in \mathbb{R}^{d},
$$

where $f: S \times \mathbb{R}^{d} \rightarrow \mathbb{R}$ is such that $f_{x}:=f(\cdot, x)$ is integrable on $S$; for such functions we refer to [8], [28]. We review the extended work of E. Giner, providing simple proofs and completing it in a general approach encompassing the crucial case of F-I-regularity ( $\mathrm{F}$ for firm or Fréchet and I for incident or intermediate or adjacent) considered in [25]. There it is shown that F-I-regularity can be transferred from an integrand satisfying a certain growth condition to the corresponding integral functional on some Lebesgue space. In contrast, it is shown in [31, Thm 4.3] that the integral functional $I_{f}$ on $L_{1}\left(S, \mathbb{R}^{d}\right)$ associated with an integrand $f$ is not $C-L$-regular at any $\bar{x} \in L_{1}\left(S, \mathbb{R}^{d}\right)$ such that $f$ is not $C-D$-regular along $\bar{x}$ (i.e. $\partial_{C} f_{s}(\bar{x}(s)) \neq \partial_{D} f_{s}(\bar{x}(s)$ ) a.e.) and produce an example showing that $C-D$-regularity of $f$ along $\bar{x}$ does not ensure that $I_{f}$ is $C-L$ regular at $\bar{x}$.

In the sequel $(S, \mathcal{S}, \mu)$ is a $\sigma$-finite complete measure space and $p \in] 1, \infty[$. The case $p=1$ and $p=\infty$ are particular enough (see [7], [31] and [43] for the case $p=1$ and [25] for the case $p=\infty$ ), so we discard them here.

\footnotetext{
${ }^{1}(*)$ Laboratoire MIP, IMT, Université Paul Sabatier, 118 route de Narbonne 31062 Toulouse cedex 04, France. emmanuel.giner@wanadoo.fr

$(\dagger)$ Laboratoire Jacques-Louis Lions, Sorbonne Univ., U.P.M.C. Paris 6, 4 place Jussieu 75005 PARIS penotj@ljll.math.upmc.fr
} 
We also give a glimpse to an analogue of the Fenchel transform which can be used for nonconvex functionals. Applying it to integral functionals offers a reminiscence of the early work of R.T. Rockafellar on convex integral functionals [47], [48]. Since here we consider nonconvex integrands and nonconvex integral functionals, we have to use general subdifferentials. Their definitions are recalled in the next section. Basic facts about integral functionals are presented in Section 3. Since calmness plays a crucial role in this study, it is characterized in Section 4. The main subdifferentials of integral functionals are investigated in Sections 5-7 and some conclusions about A-B regularity are derived in Section 8. We conclude with a section devoted to duality.

\section{Preliminaries on nonsmooth analysis}

Let us recall the definitions of the subdifferentials that will be considered here. We do not look for completeness: we do not consider the cases of the graded subdifferentials of Ioffe nor the moderate subdifferential of Michel-Penot ([45]).

For $p>1$ and a Banach space $X$ one can define a p-proximal subdifferential of a function $f: X \rightarrow \overline{\mathbb{R}}$ finite at $x$ by setting

$$
x^{*} \in \partial_{P} f(x) \Longleftrightarrow \liminf _{u \rightarrow 0} \frac{1}{\|u\|^{p}}\left[f(x+u)-f(x)-\left\langle x^{*}, u\right\rangle\right]>-\infty .
$$

The case $p=2$ is classical, but the general case is adapted to the study of functionals defined on an $L_{p}$ space.

Besides this subdifferential, the simplest one, and also the smallest one among classical subdifferentials, is the firm or Fréchet subdifferential denoted by $\partial_{F} f:$ for $f: X \rightarrow \overline{\mathbb{R}}$ finite at $x$

$$
x^{*} \in \partial_{F} f(x) \Longleftrightarrow \exists b: X \rightarrow X^{*}: x^{*}=\lim _{w \rightarrow x} b(w), \quad f(w)-f(x) \geq b(w) .(w-x) .
$$

This definition appears as a one-sided version of Fréchet differentiability. It can be shown that this simple definition is equivalent to the more usual condition

$$
x^{*} \in \partial_{F} f(x) \Longleftrightarrow \liminf _{u \rightarrow 0} \frac{1}{\|u\|}\left[f(x+u)-f(x)-\left\langle x^{*}, u\right\rangle\right] \geq 0 .
$$

In finite dimensions $\partial_{F} f(x)$ coincides with the directional (or contingent or Dini or Bouligand) subdifferential $\partial_{D} f(x)$ defined by

$$
x^{*} \in \partial_{D} f(x) \Longleftrightarrow \forall u \in X \quad f^{D}(x, u):=\liminf _{(t, v) \rightarrow\left(0_{+}, u\right)} \frac{1}{t}[f(x+t v)-f(x)] \geq\left\langle x^{*}, u\right\rangle .
$$

It can be given a geometric description in terms of the tangent cone (or contingent cone or directional tangent cone) to the epigraph $E$ of $f$ at $z:=(x, f(x))$ defined by

$$
\begin{aligned}
T(E, z) & =T^{D}(E, z)=\limsup _{t \rightarrow 0_{+}} \frac{1}{t}(E-z):=\left\{w: \liminf _{t \rightarrow 0_{+}} d\left(w, \frac{1}{t}(E-z)\right)=0\right\} \\
& =\left\{w: \exists\left(t_{n}\right) \rightarrow 0_{+},\left(w_{n}\right) \rightarrow w, z+t_{n} w_{n} \in E \forall n \in \mathbb{N}\right\} .
\end{aligned}
$$

One has $f^{D}(x, u)=\inf \{r \in \mathbb{R}:(u, r) \in T(E, z)\}$. A similar characterization can be given for the incident (or intermediate or adjacent) subdifferential in terms of the incident cone $T^{I}(E, z)$ to the epigraph $E$ of $f$ at $z:=(x, f(x))$ via the relation

$$
x^{*} \in \partial_{I} f(x) \Longleftrightarrow \forall u \in X \quad\left\langle x^{*}, u\right\rangle \leq f^{I}(x, u):=\inf \left\{r:(u, r) \in T^{I}(E, z)\right\}
$$

where $T^{I}(E, z)$ is the incident cone to $E$ at $z$, or set of velocities of moving points in $E$ :

$$
\begin{aligned}
w & \in T^{I}(E, z)=\liminf _{t \rightarrow 0_{+}} \frac{1}{t}(E-z) \Longleftrightarrow \lim _{t \rightarrow 0_{+}} \frac{1}{t} d(z+t w, E)=0 \\
& \Longleftrightarrow \exists c: \mathbb{R}_{+} \rightarrow E, c(0)=z, \quad c^{\prime}(0)=w .
\end{aligned}
$$


This last equivalence stems from the fact that when $\varepsilon(t):=(1 / t) d(z+t w, E) \rightarrow 0$ as $t \rightarrow 0_{+}$one can find $w(t) \in B(w, \varepsilon(t)+t)$ such that $c(t):=z+t w(t) \in E$ for $t>0$ small enough (or $t \in \mathbb{R}_{+}$by taking $c(t)=z$ for $t$ aloof 0$)$.

The circa-subdifferential or Clarke subdifferential $\partial_{C} f(x)$ is defined similarly

$$
x^{*} \in \partial_{C} f(x) \Longleftrightarrow \forall u \in X \quad\left\langle x^{*}, u\right\rangle \leq f^{C}(x, u):=\inf \left\{r:(u, r) \in T^{C}(E, z)\right\},
$$

where $T^{C}(E, z)$ is the circa-tangent cone or Clarke tangent cone to $E$ at $z$ :

$$
T^{C}(E, z):=\liminf _{\left(t, z^{\prime}\right)(\in \mathbb{P} \times E) \rightarrow\left(0_{+}, z\right)} \frac{1}{t}\left(E-z^{\prime}\right) .
$$

Finally, the limiting subdifferential of $f$ at $x$ is the set $\partial_{L} f(x)$ of weak* limits of sequences $\left(x_{n}^{*}\right)$ satisfying $x_{n}^{*} \in \partial_{F} f\left(x_{n}\right)$ for some $\left(x_{n}\right) \rightarrow x$ satisfying $\left(f\left(x_{n}\right)\right) \rightarrow f(x)$. For a slightly more general definition, see [33].

All these subdifferentials satisfy important common calculus rules (see [29], [44]). As an example, let us mention that for semi-separable functions one has the following rule.

Proposition 1 Let $W, X$ be normed spaces, $g: W \rightarrow \mathbb{R}_{\infty}:=\mathbb{R} \cup\{\infty\}, h: X \rightarrow \mathbb{R}_{\infty}, B \in L(W, X)$, $f: W \times X \rightarrow \mathbb{R}_{\infty}$ given by $f(w, x):=g(w)+h(B w+x)$. Then

$$
\left(w^{*}, x^{*}\right) \in \partial f(w, x) \Leftrightarrow \exists u^{*} \in \partial g(w), \exists x^{*} \in \partial h(B w+x),\left(w^{*}, x^{*}\right)=\left(u^{*}+B^{\top} x^{*}, x^{*}\right) .
$$

These subdifferentials also have different properties and it can be sensible to try to combine them, at least for some functions that are regular enough. While the "elementary subdifferentials" have nice properties in terms of order, the subdifferentials $\partial_{C}$ and $\partial_{L}$ are more efficient in terms of calculus rules. Still, the latter enjoy some order properties (see [36]) and the former dispose of some calculus rules such as

$$
\partial(f+g)(x) \subset \partial f(x) \boxminus \partial(-g)(x)
$$

where, for $A, B \subset X^{*}$ one sets $A \boxminus B:=\left\{x^{*} \in X^{*}: x^{*}+B \subset A\right\}$.

The proofs below use the following geometric properties whose proofs are simple consequences of the definitions.

Lemma 2 (a) If $A: X \rightarrow Y$ is a continuous linear map between two normed spaces and $x$ is a point of a subset $E$ of $X$, then one has

$$
\begin{aligned}
A\left(T^{I}(E, x)\right) & \subset T^{I}(A(E), A x), \\
A\left(T^{D}(E, x)\right) & \subset T^{D}(A(E), A x) .
\end{aligned}
$$

If $E:=A^{-1}(F)$ and if $A$ is open from $X$ onto $Y$ these inclusions are equalities.

(b) If $A$ is open from $E$ onto $A(E)$ at $x$, in the sense that for any sequence $\left(y_{n}\right)$ of $A(E)$ with limit $A x$ there exists a sequence $\left(x_{n}\right) \rightarrow x$ in $E$ such that $A x_{n}=y_{n}$ for all $n$, then

$$
A\left(T^{C}(E, x)\right) \subset T^{C}(A(E), A x) .
$$

If moreover $A$ is open from $X$ onto $Y$ and if $x \in E:=A^{-1}(F)$ then $A\left(T^{C}(E, x)\right)=T^{C}(F, A x)$.

Similar results are valid when $A$ is replaced with a differentiable (resp. circa-differentiable i.e. strictly differentiable) map. They imply the next composition rules.

Lemma 3 If $U, V$ are normed spaces and if $h: U \rightarrow V$ is differentiable at a point $u$ of $U$ then, for $g: V \rightarrow \overline{\mathbb{R}}$ finite at $h(u)$ and $f:=g \circ h$, for all $u^{\prime} \in U$ one has

$$
\begin{aligned}
g^{I}\left(h(u), h^{\prime}(u) u^{\prime}\right) & \leq f^{I}\left(u, u^{\prime}\right), \\
g^{D}\left(h(u), h^{\prime}(u) u^{\prime}\right) & \leq f^{D}\left(u, u^{\prime}\right),
\end{aligned}
$$

hence $h^{\prime}(u)^{\top}\left(\partial_{I} g(h(u))\right) \subset \partial_{I} f(u)$ and $h^{\prime}(u)^{\top}\left(\partial_{D} g(h(u))\right) \subset \partial_{D} f(u)$. If $U$ and $V$ are Banach spaces and if $h$ is continuously differentiable (or just circa-differentiable) at $u$ with $h^{\prime}(u)(U)=V$, equality holds. 


\section{Preliminaries about integral functionals}

In the sequel $E$ is a separable Banach space and $(S, \mathcal{S}, \mu)$ is a $\sigma$-finite complete measure space. Let us recall that the (upper) integral of a measurable function $u: S \rightarrow \overline{\mathbb{R}}$ is defined by

$$
\begin{aligned}
I(u) & :=\int u d \mu:=\inf \left\{\int_{S} y(s) d s: y \in L_{1}(S), u(\cdot) \leq y(\cdot) \text { a.e. }\right\} \\
& =\int \max (u, 0) d \mu-\int \max (-u, 0) d \mu .
\end{aligned}
$$

Since we adopt the convention $(+\infty)+(-\infty)=+\infty,(+\infty)-(+\infty)=(+\infty)+(-\infty)$, we have the following useful observation.

Lemma 4 For a measurable function $u: S \rightarrow \overline{\mathbb{R}}$ one has $I(-u)=-I(u)$ whenever $I(u)<\infty$ or $I(-u)<\infty$.

Proof. Assume that $I(u)<+\infty$. Then, for $u^{+}:=\max (u, 0)$ we have $I\left(u^{+}\right)<+\infty$. For $u^{-}:=(-u)^{+}$we have $I(u)=I\left(u^{+}\right)-I\left(u^{-}\right)$. Thus, when $I\left(u^{-}\right)$is finite we have $I(-u)=I\left(u^{-}\right)-I\left(u^{+}\right)=-I(u)$ and when $I\left(u^{-}\right)=+\infty$ we have $I(u)=-\infty$ and $I(-u)=+\infty$.

Given an integrand $f: S \times E \rightarrow \mathbb{R}_{\infty}$, setting $f \diamond x:=f(\cdot, x(\cdot))$, the associated integral functional $F:=I_{f}$ is defined by

$$
F(x):=I_{f}(x):=I(f \diamond x):=\inf \left\{\int_{S} y(s) d \mu(s): y \in L_{1}(S), y \geq f \diamond x \text { a.e. }\right\} .
$$

Throughout we assume that $f$ is a normal integrand in the sense that for a.e. $s \in S$ the function $f_{s}:=f(x, \cdot)$ is lower semicontinuous and the multimap $s \rightrightarrows E(s):=\operatorname{epi} f_{s}$ is measurable so that for all $x \in L_{p}(S, E)$ the function $f \diamond x$ is measurable, (see [27], [50], [52]). The definition of $I_{f}$ can be reformulated in geometric terms using the linear map

$$
A: L_{p}(S, E) \times L_{1}(S) \rightarrow L_{p}(S, E) \times \mathbb{R}
$$

defined by $A(x, y):=\left(x, \int_{S} y(s) d s\right)$ for $(x, y) \in L_{p}(S, E) \times L_{1}(S)$.

For a multimap $M: S \rightrightarrows E$ with nonempty values, we denote by $L_{p}(M)$ the set of measurable selections of $M$ that are in $L_{p}(S, E)$ :

$$
L_{p}(M):=\left\{x \in L_{p}(S, E): x(\cdot) \in M(\cdot) \text { a.e. }\right\} .
$$

Similarly, if $M: S \rightrightarrows E \times \mathbb{R}$ is a multimap, we denote by $L_{p, 1}(M)$ the set of measurable selections of $M$ that are in $L_{p}(S, E) \times L_{1}(S)$ :

$$
L_{p, 1}(M):=\left\{(x, y) \in L_{p}(S, E) \times L_{1}(S):(x(\cdot), y(\cdot)) \in M(\cdot) \text { a.e. }\right\} .
$$

Lemma 5 If $S$ is $\sigma$-finite, for any integrand $f$ one has

$$
\text { epi } I_{f}=A\left(L_{p, 1}(\text { epi } f)\right) \text {. }
$$

Proof. Clearly, for all $(x, y) \in L_{p, 1}$ (epi $\left.f\right)$ we have $A(x, y) \in$ epi $I_{f}$. Conversely, let $(x, r) \in$ epi $I_{f}$, so that $I(f \diamond x) \leq r$. Then, either $I(f \diamond x)=-\infty$ or $f \diamond x$ is integrable. In both cases we can find some $u \in L_{1}(S)$ satisfying $f \diamond x \leq u$ and $\int u \leq r$. Taking $z \in L_{1}(S)$ such that $z \geq 0$ and $\int z=r-\int u$ and setting $v:=u+z$, we get $(x, v) \in L_{p, 1}$ (epi $\left.f\right)$ and $A(x, v)=(x, r)$.

The following result is of crucial importance.

Lemma 6 ([27], [49], [50], [52, Thm 14.60]) Let $f: S \times E \rightarrow \overline{\mathbb{R}}$ be a normal integrand such that $\operatorname{dom} I_{f}$ is nonempty. Then

$$
\inf \left\{\int_{S} f(s, x(s)) d \mu(s): x \in L_{p}(S, E)\right\}=\int_{S} \inf _{e \in E} f(s, e) d \mu(s) .
$$

Moreover, when this common value is not $-\infty, \bar{x} \in L_{p}(S, E)$ is a minimizer of $I_{f}$ if, and only if, for a null set $N$ of $S, \bar{x}(s)$ is a minimizer of $f_{s}:=f(s, \cdot)$ for all $s \in S \backslash N$. 
An application of this result to infimal convolution is noteworthy. Here the infimal convolution of two functions $f, g: E \rightarrow \mathbb{R}_{\infty}$ is the function

$$
f \boxplus g: e \mapsto \inf \left\{f\left(e^{\prime}\right)+g\left(e^{\prime \prime}\right): e^{\prime}+e^{\prime \prime}=e\right\} .
$$

Corollary 7 Let $h, k: S \times E \rightarrow \overline{\mathbb{R}}$ be normal integrands such that $f: S \times E \rightarrow \overline{\mathbb{R}}$ defined by $f_{s}:=h_{s} \boxplus k_{s}$ is a normal integrand. Then, for $w \in \operatorname{dom} I_{f}$ one has $I_{f}(w)=\left(I_{h} \boxplus I_{k}\right)(w)$. Moreover, the infimal convolution $I_{h} \boxplus I_{k}$ is exact at $w \in \operatorname{dom} I_{f}$ if and only if for a.e. $s \in S$ the infimal convolution of $h_{s}$ and $k_{s}$ is exact.

Proof. This follows from the lemma since

$$
\left(I_{h} \boxplus I_{k}\right)(w):=\inf \left\{\int_{S}[h(s, x(s))+k(s, w(s)-x(s))] d \mu(s): x \in L_{p}(S, E)\right\}
$$

and $\inf _{e \in E}[h(s, e)+k(s, w(s)-e)]=f(s, w(s))$.

As an application, we obtain the following regularization property. In the case $p=2$, such a regularization is known as the Moreau regularization or the Moreau envelope.

Proposition 8 Let $f: S \times E \rightarrow \overline{\mathbb{R}}$ be a normal integrand and let $r>0, w \in L_{p}(S, E)$. Assume that there exists some $u \in L_{p}(S, E)$ such that $I_{f}(u)+\frac{1}{r}\|w-u\|_{p}^{p}<\infty$. Then one has

$$
\left(I_{f} \boxplus \frac{1}{r}\|\cdot\|_{p}^{p}\right)(w)=\int_{S}\left(f_{s} \boxplus \frac{1}{r}\|\cdot\|_{E}^{p}\right)(w(s)) d \mu(s) .
$$

In other terms, the regularized function of $I_{f}$ for the kernel given by the $p$-th power of the norm on $L_{p}(S, E)$ is the integral functional of the regularized integrand by the $p$-th power of the norm on $E$. In view of this proposition and of the results in [34] one can connect the subdifferentials of $I_{f}$ with the subdifferentials of the envelope of $I_{f}$, taking into account the statements of Section 8. This task is outside the scope of the present survey.

The following lemma gives the flavor of our aims. Here we use the Fenchel-Moreau subdifferential albeit $h$ is not necessarily convex. In particular, if $k: E \rightarrow \overline{\mathbb{R}}$ is positively homogeneous, we have $e^{*} \in \partial k(0)$ if and only if $\left\langle e^{*}, e\right\rangle \leq k(e)$ for all $e \in E$ and $k(0)=0$. In fact, if $e^{*} \in \partial k(0)$ we must have $k(0) \in \mathbb{R}$, hence $k(0)=0$ since $k(0) \in\{0, \infty,-\infty\}$; then $\left\langle e^{*}, e\right\rangle \leq k(e)$ for all $e \in E$. Conversely, when this property holds and $k(0)=0$ we have $e^{*} \in \partial k(0)$.

Lemma 9 Given a normal integrand $h: S \times E \rightarrow \overline{\mathbb{R}}$ that is positively homogeneous in its second variable, for $h_{s}:=h(s, \cdot)$ one has

$$
x^{*} \in \partial I_{h}(0) \Leftrightarrow x^{*} \in L_{q}(\partial h(0)):=\left\{x^{*} \in L_{q}\left(S, E^{*}\right): x^{*}(s) \in \partial h_{s}(0) \text { a.e. }\right\} .
$$

Proof. Since $I_{h}$ is positively homogeneous when $h_{s}$ is positively homogeneous for all $s \in S$, when $x^{*} \in$ $\partial I_{h}(0)$ we have $I_{h}(0)=0$, hence $0 \in \operatorname{dom} I_{f}$ for $f$ given by $f(s, e):=h(s, e)-\left\langle x^{*}(s), e\right\rangle$ and since 0 is a minimizer of $I_{f}, 0$ is a minimizer of $f_{s}$ and, for any measurable subset $A$ of $S$ we have

$$
0=\inf _{x \in L_{p}(S, E)}\left(I_{h}\left(1_{A} x\right)-\left\langle x^{*}, 1_{A} x\right\rangle\right)=\inf _{x \in L_{p}(S, E)} \int_{A}\left(h(s, x(s))-\left\langle x^{*}(s), x(s)\right\rangle\right) d \mu(s)
$$

so that $x^{*}(s) \leq h(s, \cdot)$ a.e.

Conversely, if $x^{*} \in L_{q}(\partial h(0))$ we have $h(s, 0)=0$ a.e. and the relation $x^{*}(s) \leq h(s, \cdot)$ a.e. implies that $\left\langle x^{*}, u\right\rangle \leq I_{h}(u)$ for all $u \in L_{p}(S, E)$, hence $x^{*} \in \partial I_{h}(0)$.

Let us say that an integrand $f: S \times E \rightarrow \mathbb{R}$ satisfies the growth condition $(\mathrm{G})$ if there exist $a \in L_{1}(S, \mathbb{R})$, $b \in \mathbb{R}_{+}$and a null set $N \subset S$ such that

$$
f(s, e) \geq a(s)-b\|e\|^{p} \quad \forall(s, e) \in(S \backslash N) \times E .
$$


This condition is equivalent to the following one:

$\left(\mathrm{G}^{\prime}\right)$ for all $z \in L_{p}(S, E)$ there exist some $a_{z} \in L_{1}(S, \mathbb{R}), b_{z} \in \mathbb{R}_{+}$and a null set $N \subset S$ such that

$$
\left(\mathrm{G}_{z}\right) \quad f(s, e) \geq a_{z}(s)-b_{z}\|e-z(s)\|^{p} \quad \forall(s, e) \in(S \backslash N) \times E .
$$

The equivalence is a consequence of the relation $\|e-z(s)\|^{p} \leq 2^{p-1}\|e\|^{p}+2^{p-1}\|z(s)\|^{p}$, changing $b_{z}$ into $2^{p-1} b_{z}$ and $a_{z}(s)$ into $a_{z}(s)-2^{p-1} b_{z}\|z(s)\|^{p}$ to pass from the second form to the first one, the reverse being obtained by taking $z=0$. Similarly, condition $(\mathrm{G})$ is equivalent to the existence of some $z \in L_{p}(S, E)$, $a_{z} \in L_{1}(S, \mathbb{R}), b_{z} \in \mathbb{R}_{+}$and a null set $N \subset S$ such that relation (G') holds.

Lemma 10 Let $f$ be a normal integrand satisfying condition $(G)$. Then $I_{f}$ is lower semicontinuous on its domain in $L_{p}(S, E)$.

Proof. Given $x \in L_{p}(S, E)$ and a sequence $\left(x_{n}\right) \rightarrow x$ in $L_{p}(S, E)$, we can find a subsequence $\left(x_{k(n)}\right)$ such that $\left(I_{f}\left(x_{k(n)}\right)\right) \rightarrow \liminf _{n} I_{f}\left(x_{n}\right)$ and $\left(x_{k(n)}\right) \rightarrow x$ a.e. Then Fatou's lemma ensures that

$$
\liminf _{n} \int_{S}\left[f \diamond x_{k(n)}+b\left\|x_{k(n)}\right\|^{p}-a\right] d \mu \geq \int_{S}\left[f \diamond x+b\|x\|^{p}-a\right] d \mu .
$$

Since $\left(\int_{S}\left\|x_{k(n)}\right\|^{p} d \mu\right) \rightarrow \int_{S}\|x\|^{p} d \mu$ we obtain that $\liminf { }_{n} I_{f}\left(x_{n}\right) \geq I_{f}(x)$.

Integral functionals enjoy some properties of convex functions.

Proposition 11 ([2, Prop. 2]) If $(S, \mathcal{S}, \mu)$ has no atom and if $f$ is convex in its second variable, $I_{f}$ is continuous on the whole of $L_{p}(S, E)$ whenever it is continuous at some point of $L_{p}(S, E)$.

Proposition 12 ([21]) If $(S, \mathcal{S}, \mu)$ has no atom and if $f$ is a (possibly nonconvex in its second variable) normal integrand, then $I_{f}$ is Lipschitzian on bounded sets whenever it is Lipschitzian around some point of $L_{p}(S, E)$.

Proposition 13 ([16]) If $(S, \mathcal{S}, \mu)$ has no atom and if $f$ is a (possibly nonconvex in its second variable) normal integrand, then any local minimizer of $I_{f}$ is a global minimizer.

We also have a kind of duality result. We give a simplified version which is sufficient for our needs, but the result holds for a finite set of integral constraints (see [4, Cor. 5.7] and its references). Such a result evokes a duality result in terms of the Lagrangian $\ell_{s}(e, y):=j_{s}(e)+y g_{s}(e)$.

Proposition 14 Let $g: S \times E \rightarrow \mathbb{R}, j: S \times E \rightarrow \overline{\mathbb{R}}$ be measurable integrands and let $b, m \in \mathbb{R}, p \in[1, \infty[$. Assume there exists some $u_{0} \in L_{p}(S, E)$ such that $I_{j}\left(u_{0}\right)<+\infty$ and $I_{g}\left(u_{0}\right) \leq 0$. Then the following assertions are equivalent:

(a) $I_{j}(u) \geq m$ for all $u \in L_{p}(S, E)$ satisfying $-\infty<I_{g}(u) \leq 0$;

(b) there exists $y \in \mathbb{R}_{+}$and $a \in L_{1}(S)$ such that $I(a) \geq m$ and $j(s, e)+y g(s, e) \geq a(s)$ for all $e \in E$ and a.e. $s \in S$.

Corollary 15 When $(S, \mathcal{S}, \mu)$ is $\sigma$-finite, under the assumptions of the proposition, given $b, m \in \mathbb{R}$ the following assertions are equivalent:

(a) $-\infty<I_{g}(u) \leq b \Longrightarrow I_{-j}(u) \leq m$ for all $u \in L_{p}(S, E)$;

(b) there exists $y \in \mathbb{R}_{+}$and $a \in \bar{L}_{1}(S)$ such that $I(a) \geq y b-m$ and $j(s, e)+y g(s, e) \geq a(s)$ for all $e \in E$ and a.e. $s \in S$.

Using Lemma 4, the implication $(\mathrm{a}) \Rightarrow(\mathrm{b})$ of the corollary is a consequence of the corresponding implication of Proposition 14 by changing $m$ into $-m$ and $g$ into $g-b v$, where $v \in L_{1}(S)$ is nonnegative and $\int_{S} v=1$. The reverse implication is immediate. 


\section{Calmness}

Let us recall a classical result showing that calmness as defined in the following statement can be formulated in various equivalent ways.

Lemma 16 (Nemeth) For $F: X \rightarrow \overline{\mathbb{R}}$ finite at $x$ in a normed space $X$ the following conditions are equivalent and are satisfied whenever $\partial_{D} F(x)$ is nonempty:

(a) $F$ is calm at $x$ : there exist $r \in \mathbb{P}:=] 0, \infty\left[\right.$ and $c \in \mathbb{R}_{+}$such that

$$
\forall w \in B(x, r) \quad F(w)-F(x) \geq-c\|w-x\| ;
$$

(b) there exists $c \in \mathbb{R}_{+}$such that $F^{D}(x, v) \geq-c\|v\|$ for all $v \in X$;

(c) for all $v \in X$ one has $F^{D}(x, v)>-\infty$;

(d) one has $F^{D}(x, 0)=0$.

Proof. The implications (a) $\Rightarrow(\mathrm{b}) \Rightarrow(\mathrm{c}) \Rightarrow(\mathrm{d})$ are obvious. Suppose (a) does not hold: there exists a sequence $\left(x_{n}\right)$ such that $r_{n}:=\left\|x_{n}-x\right\| \leq n^{-2}$ and $F\left(x_{n}\right)-F(x) \leq-n^{2} r_{n}$. Then, setting $t_{n}:=n r_{n}$ and $v_{n}:=$ $t_{n}^{-1}\left(x_{n}-x\right)$ one has $\left(v_{n}\right) \rightarrow 0$ and $t_{n}^{-1}\left(F\left(x+t_{n} v_{n}\right)-F(x)\right) \leq-n$, so that $F^{D}(x, 0)=-\infty$ and (d) does not hold.

If $\partial_{D} F(x)$ is nonempty we have $F^{D}(x, 0) \geq\left\langle x^{*}, 0\right\rangle=0$ for any $x^{*} \in \partial_{D} F(x)$ and since $F^{D}(x, 0) \in\{0,-\infty\}$, we have $F^{D}(x, 0)=0$.

For the study of the directional subdifferential of an integral functional we need the following observation required by the fact that in general $I_{-f}$ does not coincides with $-I_{f}$.

Lemma 17 Let $f: S \times E \rightarrow \overline{\mathbb{R}}$ be a measurable integrand and let $x \in L_{p}(S, E)$ be such that $f \diamond x$ is integrable. Then $I_{f}$ is calm at $x$ if, and only if $I_{-f}$ is quiet at $x$ in the sense that $J_{f}:=-I_{-f}$ is calm at $x$.

Proof. Since $J_{f} \leq I_{f}$ and $J_{f}(x)=I_{f}(x), I_{f}$ is calm at $x$ whenever $J_{f}$ is calm at $x$. Let us prove the converse. Assume $I_{f}$ is calm at $x$, so that there exist $c>0$ and $r>0$ such that

$$
I_{f}(w)-I_{f}(x) \geq-c\|w-x\|_{p} \quad \forall w \in L_{p}(S, E) \text { such that }\|w-x\|_{p}<r .
$$

Without loss of generality we assume that $x=0$ and $f \diamond x=0$. Given $w \in L_{p}(S, E)$ such that $\|w-x\|_{p}<r$, let $S_{w}:=\{s \in S: f(s, w(s)) \leq 0\}$. Then $\left\|1_{S_{w}} w\right\|_{p}<r$ and $-(f \diamond w)^{-}:=\inf (f \diamond w, 0)=1_{S_{w}}(f \diamond w)=f \diamond\left(1_{S_{w}} w\right)$, so that relation (2) yields

$$
\int_{S}-(f \diamond w)^{-}=\int_{S} f \diamond\left(1_{S_{w}} w\right) \geq-c\left\|1_{S_{w}} w\right\|_{p}>-c r .
$$

It follows that $(f \diamond w)^{-}$is integrable, hence that $J\left((f \diamond w)^{-}\right)=I\left((f \diamond w)^{-}\right)$and

$$
J_{f}(w) \geq J\left((f \diamond w)^{-}\right)=I\left((f \diamond w)^{-}\right) \geq-c\left\|1_{S_{w}} w\right\|_{p} \geq-c\|w\|_{p} .
$$

Thus $J_{f}$ is calm at $x=0$.

For a function $F: X \rightarrow \mathbb{R}_{\infty}$ on a normed space $X$, a sufficient condition for calmness at $x \in \operatorname{dom} F$ is given in the following lemma.

Lemma 18 For a function $F: X \rightarrow \mathbb{R}_{\infty}$ on a normed space $X$, a sufficient condition for calmness at $x \in \operatorname{dom} F$ is the existence of some $c_{1}$ and $c_{p}$ in $\mathbb{R}_{+}, p \in[1, \infty[$ such that

$$
\left(D_{F}\right) \quad F(x+u)-F(x) \geq-c_{1}\|u\|-c_{p}\|u\|^{p} \quad \forall u \in X .
$$

Conversely, if $F$ satisfies the growth condition

$$
\left(G_{F}\right) \quad \text { there exist } a, b \in \mathbb{R}_{+} \text {such that } F(\cdot) \geq a-b\|\cdot\|^{p}
$$

calmness of $F$ at $x \in \operatorname{dom} F$ entails condition $\left(D_{F}\right)$. 
Proof. Since for $u \in r B_{X}$ we have $F(x+u)-F(x) \geq-\left(c_{1}+c_{p} r^{p-1}\right)\|u\|$, condition $\left(\mathrm{D}_{F}\right)$ implies calmness of $F$ at $x$.

Conversely, if $F$ is calm at $x$ and if $c \in \mathbb{R}_{+}, r \in \mathbb{P}$ are such that

$$
F(x+u)-F(x) \geq-c\|u\| \quad \forall u \in r B_{X},
$$

then, under the following growth condition $\left(\mathrm{G}_{x}\right)$ in which $b_{0}, b_{p} \in \mathbb{R}_{+}$,

$$
\left(\mathrm{G}_{x}\right) \quad F(v) \geq-b_{0}-b_{p}\|v-x\|^{p} \quad \forall v \in X,
$$

setting $v:=x+u$, we see that $\left(\mathrm{D}_{F}\right)$ is satisfied with $c_{p}=b_{p}, c_{1}=\max \left(r^{-1}\left(b_{0}+F(x)\right)^{+}, c\right)$. Since condition $\left(\mathrm{G}_{F}\right)$ is equivalent to $\left(\mathrm{G}_{x}\right)$, the converse assertion is established.

Thus, the integral functional $I_{f}$ associated to an integrand $f: S \times E \rightarrow \mathbb{R}_{\infty}$ is calm at $x \in L_{p}(S, E)$ whenever there exist $a \in L_{q}(S), b \in \mathbb{R}_{+}$and a null set $N$ such that

$$
\left(\mathrm{D}_{x}\right) \quad f_{s}(x(s)+e)-f_{s}(x(s)) \geq-a(s)\|e\|-b\|e\|^{p} \quad \forall(s, e) \in(S \backslash N) \times E .
$$

That follows from Hölder's inequality.

For $p=1$, condition (4) is a necessary and sufficient condition for calmness of $F:=I_{f}$ at $x$ : the necessity of this condition is an easy consequence of Lemma 6 (see [21, Cor. 3.7]). It is a global calmness condition. As in [7], [43], we see that the case $p=1$ is a special case.

The following characterization of calmness on $L_{p}(S, E)$ for $p>1$ is more subtle.

Theorem 19 ([21]) Suppose $(S, \mathcal{S}, \mu)$ has no atom and $f: S \times E \rightarrow \overline{\mathbb{R}}$ is a measurable integrand. Let $x \in L_{p}(S, E)$ be such that $f \diamond x:=f(\cdot, x(\cdot)) \in L_{1}(S)$. Then the following assertions are equivalent:

(a) $F:=I_{f}$ is calm at $x$ on $X:=L_{p}(S, E)$;

(b) the integrand $f$ is $p$-calm at $x$ in the sense that

$\left(C_{p}\right)$ there exist $c>0$, a null set $N$ of $S$, an interval $\left.\left.R:=\right] 0, \bar{r}\right]$ with $\bar{r}>0$ and families $\left(y_{r}\right)_{r \in R}$ in $\mathbb{R}_{+},\left(a_{r}\right)_{r \in R}$ in $L_{1}(S)$ such that $I\left(a_{r}\right) \geq y_{r} r^{p}-c r$ and $f(s, x(s)+e)-f(s, x(s)) \geq a_{r}(s)-y_{r}\|e\|^{p}$ for all $(r, s, e) \in R \times(S \backslash N) \times E$.

When $(S, \mathcal{S}, \mu)$ is $\sigma$-finite, condition $\left(C_{p}\right)$ can be written:

$\left(C_{p}^{\prime}\right)$ there exist $c>0$, a null set $N$ of $S$, an interval $\left.\left.R:=\right] 0, \bar{r}\right]$ with $\bar{r}>0$ and families $\left(y_{r}\right)_{r \in R}$ in $\mathbb{R}_{+}$, $\left(b_{r}\right)_{r \in R}$ in $L_{1}(S), z \in L_{1}(S), z \geq 0$ such that $I(z)=1, I\left(b_{r}\right) \geq-c$ and $f(s, x(s)+e)-f(s, x(s))+y_{r}\|e\|^{p} \geq$ $r b_{r}(s)+r^{p} y_{r} z(s)$ for all $(r, s, e) \in R \times(S \backslash N) \times E$.

Proof. The $p$-calmness condition $\left(\mathrm{C}_{p}\right)$ is sufficient to ensure calmness of $F$ at $x$ since by Hölder's inequality it implies that for $u \in L_{p}(S, E)$ satisfying $\|u\|_{p}=r \leq \bar{r}$ one has

$$
I_{f}(x+u)-I_{f}(x) \geq I\left(a_{r}\right)-y_{r} r^{p} \geq-c r=-c\|u\|_{p} .
$$

Let us prove that $\left(\mathrm{C}_{p}\right)$ is necessary. Let us assume that for some $\left.c, \bar{r} \in \mathbb{P}:=\right] 0, \infty\left[\right.$ we have $I_{f}(x+u)-$ $I_{f}(x) \geq-c\|u\|_{p}$ for all $u \in \bar{r} B_{X}$. In Corollary 15, let us define integrands $g$ and $j$ by $g(s, e):=\|e\|^{p}$, $j(s, e):=f(s, x(s)+e)-f(s, x(s))$ and set $b:=r^{p}$ with $\left.\left.r \in R:=\right] 0, \bar{r}\right], m:=c r$, so that, by Lemma 17 ,

$$
I_{g}(u) \leq r^{p} \Longrightarrow I_{-j}(u) \leq c r .
$$

Corollary 15 yields $y_{r} \in \mathbb{R}_{+}, a_{r} \in L_{1}(S)$, and a null set $N$ of $S$ such that $I\left(a_{r}\right) \geq y_{r} r^{p}-c r$ and $a_{r}(s) \leq$ $j(s, e)+y_{r} g(s, e)$ for all $(s, e) \in(S \backslash N) \times E$. That means that

$$
f(s, x(s)+e)-f(s, x(s)) \geq a_{r}(s)-y_{r}\|e\|^{p} \quad \text { for all }(r, s, e) \in R \times(S \backslash N) \times E .
$$

Thus $\left(\mathrm{C}_{p}\right)$ is satisfied.

When $(S, S, \mu)$ is $\sigma$-finite we can pick $z \in L_{1}(S)$ such that $I(z)=1$ and $z \geq 0$. It suffices to set $b_{r}:=$ $r^{-1} a_{r}-r^{p-1} y_{r} z$ to obtain $\left(\mathrm{C}_{p}^{\prime}\right)$. Conversely, when $\left(\mathrm{C}_{p}^{\prime}\right)$ holds, setting $a_{r}:=r b_{r}+r^{p-1} y_{r} z$ we obtain $\left(\mathrm{C}_{p}\right)$. $\square$ 
Remark. Since condition (D) implies calmness of $I_{f}$, one may guess that it implies $\left(\mathrm{C}_{p}\right)$. That is the case, as one can see by using the inequality $a(s)\|e\| \leq \frac{1}{q}|a(s)|^{q}+\frac{1}{p}\|e\|^{p}$, taking $\bar{r}=1, a_{r}(s):=-\frac{1}{q}|a(s)|^{q}, y_{r}:=b+\frac{1}{p}$, since for $c=\min \left(\frac{1}{q}\|a\|_{q}^{q}, b+\frac{1}{p}\right)$ one has $I\left(a_{r}\right) \geq y_{r} r^{p}-c r$ for $\left.\left.r \in\right] 0,1\right]$.

On the other hand, the following criterion entails condition $\left(\mathrm{D}_{F}\right)$, hence $p$-calmness. It is slightly more general than a criterion in [25] since it involves the Fréchet subdifferential rather than the Clarke subdifferential; on the other hand it is restricted to the class of Asplund spaces.

Criterion 20 If $E$ is an Asplund space (resp. a general Banach space) and if the following condition holds, then condition $(D)$ is satisfied at $x \in \operatorname{dom} I_{f}$ : there exist $a \in L_{q}(S, \mathbb{R})$, a null set $N$, and $c \in \mathbb{R}_{+}$such that for all $(s, e) \in(S \backslash N) \times E, e^{*} \in \partial_{F} f_{s}(e)\left(r e s p . e^{*} \in \partial_{C} f_{s}(e)\right)$ one has

$$
\left\|e^{*}\right\| \leq a(s)+c\|e\|^{p-1} .
$$

The proof is an easy consequence of the Mean Value Inequality ([45, Thm 4.89]).

\section{The case of the firm subdifferential}

We need a preliminary result about Nemytskii operators in a form slightly more precise than the classical result of Krasnoselskii (see [32], [46]).

Let us consider the following growth condition in which $p, q \in] 1, \infty[, E, F$ are Banach spaces and $g$ : $S \times E \rightarrow F$ is a measurable map:

$(\mathrm{N})$ there exist $a \in \mathcal{L}_{q}(S, \mathbb{R}), z \in \mathcal{L}_{p}(S, E), b \in \mathbb{R}_{+}$and a null set $N \subset S$ such that

$$
\forall(s, e) \in(S \backslash N) \times E \quad\|g(s, e)\| \leq a(s)+b\|e-z(s)\|^{p / q} .
$$

Proposition 21 For a measurable map $g: S \times E \rightarrow F$ satisfying the growth condition (N) the following assertions hold:

(a) for all $u \in L_{p}(S, E)$ the map $v:=g \diamond u:=g(\cdot, u(\cdot))$ belongs to $L_{q}(S, F)$;

(b) if for some $\bar{u} \in L_{p}(S, E)$ and all $s \in S \backslash N$ the map $g_{s}:=g(s, \cdot)$ is continuous at $\bar{u}(s)$, then the Nemytskii map $G: L_{p}(S, E) \rightarrow L_{q}(S, F)$ given by $G(u)=g(\cdot, u(\cdot))$ is continuous at $\bar{u}$.

Proof. Condition (N) implies that $a$ is nonnegative. Clearly, for $u \in L_{p}(S, E)$ the map $v:=g \diamond u:=g(\cdot, u(\cdot))$ is measurable and satisfies for $s \in S \backslash N$

$$
\|g(s, u(s))\|^{q} \leq\left|a(s)+b\|u(s)-z(s)\|^{p / q}\right|^{q} \leq 2^{q-1} a(s)^{q}+2^{q-1} b^{q}\|u(s)-z(s)\|^{p} .
$$

Integrating, for $v:=g \diamond u:=g(\cdot, u(\cdot))$, we get

$$
\int_{S}\|v(s)\|^{q} d \mu(s) \leq 2^{q-1}\|a\|_{q}^{q}+2^{q-1} b^{q}\|u-z\|_{p}^{p}<\infty
$$

so that $v \in L_{q}(S, F)$.

Given $\bar{u} \in L_{p}(S, E)$ and a sequence $\left(u_{n}\right) \rightarrow \bar{u}$ in $L_{p}(S, E)$, we pick a subsequence $\left(u_{k(n)}\right)$ such that $\left\|u_{k(n+1)}-u_{k(n)}\right\| \leq 2^{-n}$. Then $\left(u_{n}\right) \rightarrow \bar{u}$ a.e. and there exists some $h \in L_{p}(S, \mathbb{R})$ such that $\left\|u_{k(n)}(s)-z(s)\right\| \leq$ $h(s)$ a.e. for all $n$ (see [46]). Using assumption (N), we get

$$
\begin{aligned}
\left\|g\left(s, u_{n}(s)\right)-g(s, \bar{u}(s))\right\|^{q} & \leq 2^{q-1}\left\|g\left(s, u_{n}(s)\right)\right\|^{q}+2^{q-1}\|g(s, \bar{u}(s))\|^{q} \\
& \leq 2^{q-1}\left(2^{q} a(s)^{q}+2^{q-1} b^{q}\left(\left\|u_{n}(s)-z(s)\right\|^{p}+\|\bar{u}(s)-z(s)\|^{p}\right)\right) \\
& \leq 2^{2 q-1} a(s)^{q}+2^{2 q-1} b^{q} h(s)^{p} .
\end{aligned}
$$

Observing that $\left(g\left(s, u_{n}(s)\right)\right) \rightarrow g(s, \bar{u}(s))$ a.e. and applying the dominated convergence theorem, we obtain that $\left(g \diamond u_{k(n)}\right) \rightarrow g \diamond \bar{u}$ in $L_{q}(S, F)$. Since $\left(u_{n}\right)$ is any sequence converging to $\bar{u}$, we obtain that $G$ is continuous at $\bar{u}$. 
Theorem 22 Suppose that for $p \in] 1, \infty\left[, x \in L_{p}(S, E)\right.$, the integrand $f$ satisfies condition $\left(D_{F}\right)$ in relation (4). Then, setting $q:=(1-1 / p)^{-1}$, denoting by $L_{q}\left(\partial_{F} f \diamond x\right)$ the set of selections $x^{*} \in L_{q}\left(S, E^{*}\right)$ of the multimap $s \rightrightarrows \partial_{F} f_{s}(x(s))$, one has the inclusion

$$
L_{q}\left(\partial_{F} f \diamond x\right) \subset \partial_{F} I_{f}(x) .
$$

For the case $p=1$ we refer to [7] and [43].

Proof. Let $x^{*} \in L_{q}\left(\partial_{F} f \diamond x\right)$ and let $g: S \times E \rightarrow \mathbb{R}$ be given by

$$
g(s, e)=-\frac{1}{\|e\|}\left(f(x(s)+e)-f(x(s))-\left\langle x^{*}(s), e\right\rangle\right)
$$

for $(s, e) \in S \times(E \backslash\{0\}), g(s, 0)=0$. Considering the sets $\{(s, e): g(s, e)<r\}$, one sees that $g$ and $g^{+}:=\max (g, 0)$ are measurable. Moreover, by condition (4), one has

$$
g(s, e) \leq|a(s)|+\left\|x^{*}(s)\right\|+b\|e\|^{p-1} \quad \forall(s, e) \in(S \backslash N) \times E .
$$

Since $p-1=p / q$ and since $x^{*} \in L_{q}\left(S, E^{*}\right)$, we see that $g^{+}$satisfies condition (N) with $F:=\mathbb{R}, z=0$, and $a$ changed into $|a|+\left\|x^{*}(\cdot)\right\|$. Proposition 21 and the fact that $g^{+}(s, e) \rightarrow 0$ as $e \rightarrow 0$ ensure that $g^{+} \diamond u \rightarrow 0$ in $L_{q}(S, \mathbb{R})$ as $u \rightarrow 0$ in $L_{p}(S, E)$. Since

$$
I_{f}(x+u)-I_{f}(x)-\left\langle x^{*}, u\right\rangle \geq-\int g^{+}(s, u(s))\|u(s)\| d \mu(s) \geq-\left\|g^{+} \diamond u\right\|_{q}\|u\|_{p}
$$

that shows that $x^{*} \in \partial_{F} I_{f}(x)$.

One may wonder whether one can drop assumption $\left(D_{F}\right)$ in the preceding statement. The following counter-example shows that it is not the case.

Counter-example. Let $S:=[0,1]$ with its Lebesgue measure and for $n \geq 1$ let $S_{n}:=\left[0, n^{-4}\right]$. Consider the integrand $f: S \times \mathbb{R} \rightarrow \mathbb{R}$ given by $f(s, e):=e^{3}$ and the associated integral functional $I_{f}$ on $L_{2}(S, \mathbb{R})$. For all $s \in S$ one has $\partial_{F} f_{s}(0)=\{0\}$. Thus, for $x:=0$ we have $L_{2}\left(\partial_{F} f \diamond x\right)=\{0\}$. However we have $\partial_{F} I_{f}(x)=\varnothing$ since for $x_{n}:=-n 1_{S_{n}}$ we have $\left\|x_{n}\right\|_{2}=1 / n$ but $I_{f}\left(x_{n}\right) /\left\|x_{n}\right\|_{2}=-1$, so that for $x^{*}=0$ one has $\lim _{n}\left(1 /\left\|x_{n}\right\|_{2}\right)\left(I_{f}\left(x_{n}\right)-I_{f}(0)-\left\langle x^{*}, x_{n}\right\rangle\right)=-1$.

\section{Subderivatives of integral functionals}

In this section we consider generalized directional derivatives (subderivatives in the terminology of [52]) as well as subdifferentials.

\subsection{The case of the incident subdifferential}

The following result is remarkable because it does not require any restrictive assumption.

Theorem 23 ([15], [25]) Let $f: S \times E \rightarrow \mathbb{R}_{\infty}:=\mathbb{R} \cup\{\infty\}$ be a normal integrand finite at $x \in L_{p}(S, E)$. Let $h: S \times E \rightarrow \overline{\mathbb{R}}$ be the integrand given by $h(s, e):=f_{s}^{I}(x(s), e)$ for $(s, e) \in S \times E$, for $f_{s}:=f(s, \cdot)$. Then, for all $u \in L_{p}(S, E)$ one has $\left(I_{f}\right)^{I}(x, u) \leq I_{h}(u)$ or

$$
\begin{gathered}
\left(I_{f}\right)^{I}(x, u) \leq I\left(f^{I} \diamond(x, u)\right), \\
\partial_{I} I_{f}(x) \subset L_{q}\left(\partial_{I} f \diamond x\right),
\end{gathered}
$$

where $q:=(1-1 / p)^{-1}$ and where $L_{q}\left(\partial_{I} f \diamond x\right)$ is the set of $x^{*} \in L_{q}\left(S, E^{*}\right)$ such that $x^{*}(s) \in \partial_{I} f_{s}(x(s))$ a.e..

Its proof relies on the following result of independent interest. 
Proposition 24 Given a measurable multimap $M: S \rightrightarrows E \times \mathbb{R}, z:=(x, y) \in L_{p, 1}(M)$, let $T_{z}^{I} M$ be the multimap $s \rightrightarrows T^{I}(M(s), z(s))$ and let $T_{z}^{D} M$ be the multimap $s \rightrightarrows T^{D}(M(s), z(s))$. Then the following inclusions hold:

$$
\begin{aligned}
L_{p, 1}\left(T_{z}^{I} M\right) & \subset T^{I}\left(L_{p, 1}(M), z\right) \\
T^{D}\left(L_{p, 1}(M), z\right) & \subset L_{p, 1}\left(T_{z}^{D} M\right) .
\end{aligned}
$$

Proof. Let us define $d:(E \times \mathbb{R}) \times(E \times \mathbb{R}) \rightarrow \mathbb{R}$ by

$$
d\left((e, r),\left(e^{\prime}, r^{\prime}\right)\right):=\left\|e-e^{\prime}\right\|^{p}+\left|r-r^{\prime}\right| .
$$

If $C$ is a nonempty subset of $E \times \mathbb{R}$, let $d((e, r), C):=\inf _{\left(e^{\prime}, r^{\prime}\right) \in C} d\left((e, r),\left(e^{\prime}, r^{\prime}\right)\right)$. Then, since $\left(\left(e_{n}, r_{n}\right)\right) \rightarrow(e, r)$ if, and only if $\left(d\left((e, r),\left(e_{n}, r_{n}\right)\right) \rightarrow 0\right.$, given $(a, b) \in C,(e, r) \in E \times \mathbb{R}$ we have

$$
(e, r) \in T^{I}(C,(a, b)) \Longleftrightarrow\left(d\left((e, r), t^{-1}(C-(a, b))\right) \rightarrow 0 \quad \text { as } t \rightarrow 0_{+} .\right.
$$

Similarly, we define $d_{p, 1}:\left(L_{p}(S, E) \times L_{1}(S)\right) \times\left(L_{p}(S, E) \times L_{1}(S)\right) \rightarrow \mathbb{R}$ by

$$
d_{p, 1}\left((u, v),\left(u^{\prime}, v^{\prime}\right)\right)=\int_{S}\left(\left\|u(s)-u^{\prime}(s)\right\|^{p}+\left|v(s)-v^{\prime}(s)\right|\right) d \mu(s) .
$$

Then, for $z:=(x, y) \in L_{p, 1}(M)$ we have

$$
w:=(u, v) \in T^{I}\left(L_{p, 1}(M), z\right) \Longleftrightarrow\left(d_{p, 1}\left((u, v), \frac{1}{t}\left[L_{p, 1}(M)-(x, y)\right]\right) \rightarrow 0 \quad \text { as } t \rightarrow 0_{+} .\right.
$$

Moreover, setting for $t>0,(s, e, r) \in S \times E \times \mathbb{R}$

$$
g_{t}(s, e, r):=d\left((u(s), v(s)), t^{-1}((e, r)-(x(s), y(s)))\right)+\iota_{M(s)}(e, r),
$$

we see that

$$
d_{p, 1}\left((u, v), \frac{1}{t}\left[L_{p, 1}(M)-(x, y)\right]\right)=\inf \left\{\int_{S} g_{t}\left(s, u^{\prime}(s), v^{\prime}(s)\right):\left(u^{\prime}, v^{\prime}\right) \in L_{p}(S, E) \times L_{1}(S)\right\} .
$$

Using a variant of Proposition 6 and noting that since $(0,0) \in t^{-1}\left[L_{p, 1}(M)-(x, y)\right]$ we have

$$
d_{p, 1}\left((u, v), \frac{1}{t}\left[L_{p, 1}(M)-(x, y)\right]\right) \leq\|u\|_{p}^{p}+\|v\|_{1}
$$

we obtain

$$
d_{p, 1}\left((u, v), t^{-1}\left(L_{p, 1}(M)-(x, y)\right)=\int_{S} \inf _{(e, r) \in E \times \mathbb{R}} g_{t}(s, e, r) d \mu(s) .\right.
$$

Given $(u, v) \in L_{p, 1}\left(T_{z}^{I} M\right)$ we have $\lim _{t \rightarrow 0_{+}} g_{t}(s, u(s), v(s))=0$ a.e. Then, for any sequence $\left(t_{n}\right) \rightarrow 0_{+}$we have

$$
d_{p, 1}\left((u, v), t_{n}^{-1}\left[L_{p, 1}(M)-(x, y)\right]\right) \leq \int_{S} g_{t_{n}}(s, u(s), v(s)) d \mu(s) .
$$

Since for all $n \in \mathbb{N}$ we have $g_{t_{n}}(s, u(s), v(s)) \leq\|u(s)\|^{p}+|v(s)|$ for all $s \in S$, and since $\left(g_{t_{n}}(s, u(s), v(s))\right) \rightarrow 0$ as $n \rightarrow \infty$, the dominated convergence theorem yields $\left(d_{p, 1}\left((u, v), t_{n}^{-1}\left(L_{p, 1}(M)-(x, y)\right)_{n} \rightarrow 0\right.\right.$. That shows that $(u, v) \in T^{I}\left(L_{p, 1}(M), z\right)$.

Given $(v, w) \in T^{D}\left(L_{p, 1}(M), z\right)$ there exist sequences $\left(t_{n}\right) \rightarrow 0_{+},\left(\left(v_{n}, w_{n}\right)\right) \rightarrow(v, w)$ in $L_{p, 1}(E \times \mathbb{R})$ such that $(x, y)+t_{n}\left(v_{n}, w_{n}\right) \in L_{p, 1}(M)$ for all $n \in \mathbb{N}$. That means that for all $n \in \mathbb{N}$ there exists a null subset $N_{n}$ of $S$ such that $\left(x(s)+t_{n} v_{n}(s), y(s)+y_{n} w_{n}(s)\right) \in M(s)$ for all $s \in S \backslash N_{n}$. The set $N:=\cup_{n} N_{n}$ is a null set and there exist a null set $N^{\prime}$ and a subsequence $\left(\left(v_{k(n)}, w_{k(n)}\right)\right)$ of $\left(\left(v_{n}, w_{n}\right)\right)$ such that $\left(\left(v_{k(n)}(s), w_{k(n)}(s)\right)\right) \rightarrow$ $(v(s), w(s))$ a.e.for all $s \in S \backslash N^{\prime}$. Thus, for $s \in S \backslash\left(N \cup N^{\prime}\right)$ one has $(v(s), w(s)) \in T^{D}(M(s), z(s))$. Therefore $(v, w) \in L_{p, 1}\left(T_{z}^{D} M\right)$. 
Proof of Theorem 23. (a) Relation (5) is equivalent to epi $I_{h} \subset$ epi $I_{f}^{I}(x, \cdot)$. This inclusion is a consequence of the following relations in which $y(s):=f(s, x(s)), z(s):=(x(s), y(s)), r:=I_{f}(x)$, and $T^{I}$ (epi $\left.f, z\right)$ denotes the multimap $s \rightrightarrows T^{I}\left(\right.$ epi $\left.f_{s}, z(s)\right)$ :

$$
\text { epi } \begin{aligned}
I_{h} & =A\left(L_{p, 1}(\text { epi } h)\right) & & (\text { Lemma } 5) \\
& =A\left(L_{p, 1}\left(T^{I}(\text { epi } f, z)\right)\right) & & \left(\text { definition of } h:=f^{I}(x(\cdot), \cdot)\right) \\
& \subset A\left(T^{I}\left(L_{p, 1}(\text { epi } f), z\right)\right) & & (\text { Proposition 24) } \\
& \subset T^{I}\left(A\left(L_{p, 1}(\text { epi } f), A z\right)\right. & & (\text { Lemma 2) } \\
& =T^{I}\left(\text { epi } I_{f},(x, r)\right) & & \text { Lemma } 5) .
\end{aligned}
$$

(b) It is a consequence of Lemma 9 and of the definition of $\partial_{I} I_{f}$.

\subsection{The case of the circa-subdifferential}

A similar approach can be conducted for the Clarke (or circa-) subdifferential, even if the integrand is not locally Lipschitzian. However, we need the growth assumption $(\mathrm{G})$ to show that the openness property of Lemma 2 is satisfied by the map $A$ described in relation (1).

Proposition 25 ([17]) Assume $(S, \mathcal{S}, \mu)$ is $\sigma$-finite. Let $A: L_{p}(S, E) \times L_{1}(S) \rightarrow L_{p}(S, E) \times \mathbb{R}$ be given by $A(x, y):=\left(x, \int_{S} y(s) d s\right)$ for $(x, y) \in L_{p}(S, E) \times L_{1}(S)$ and let $f: S \times E \rightarrow \mathbb{R}_{\infty}$ be a normal integrand satisfying condition $(G)$. Then, for all $x \in \operatorname{dom} I_{f} \subset L_{p}(S, E), A$ is open from $L_{p, 1}($ epi $f)$ onto epi $I_{f}$ at $(x, f \diamond x)$.

Proof. Let $\left(\left(x_{n}, r_{n}\right)\right)$ be a sequence in epi $I_{f}$ with limit $(x, r)$, with $r \geq I(f \diamond x)$. We want to prove that there exists a sequence $\left(y_{n}\right)$ in $L_{1}(S)$ with limit $y:=f \diamond x$ such that $y_{n} \geq f \diamond x_{n}$ and $\int y_{n}=r_{n}$ for all $n$. Let first do that when $r=I(f \diamond x)$. Without loss of generality, taking a subsequence if necessary, we may assume that $\left(x_{n}\right) \rightarrow x$ a.e. and that there exists some $h \in L_{p}(S)$ such that $\left\|x_{n}(s)\right\|^{p} \leq h(s)$ a.e. Lemma 5 yields a sequence $\left(u_{n}\right)$ in $L_{1}(S)$ such that $r_{n}=I\left(u_{n}\right)$ and $u_{n} \geq f \diamond x_{n}$ for all $n$. Since $f$ is a normal integrand, the sequence $\left(v_{n}\right):=\left(\min \left(u_{n}, f \diamond x\right)\right)$ converges to $y:=f \diamond x$ a.e.. By condition $(\mathrm{G})$ for some $a \in L_{1}(S), b \in \mathbb{R}_{+}$ we have

$$
u_{n} \geq f \diamond x_{n} \geq a(s)-b\left\|x_{n}(s)\right\|^{p} \geq a(s)-b h(s) .
$$

Since $v_{n}(s) \leq y(s):=(f \diamond x)(s)$ we get that

$$
\left|v_{n}(s)\right| \leq \max (|y(s)|,|a(s)|+b h(s))
$$

and the dominated convergence theorem ensures that $\left(v_{n}\right) \rightarrow y$ in $L_{1}(S)$. Since $u_{n}-v_{n} \geq 0$ and $\left(\int\left(u_{n}-v_{n}\right)\right)=$ $\left(r_{n}-\int v_{n}\right) \rightarrow r-\int y=0$ we have $\left(u_{n}-v_{n}\right) \rightarrow 0$ in $L_{1}(S)$, hence $\left(u_{n}\right) \rightarrow y$ in $L_{1}(S)$. Thus we can take $y_{n}:=u_{n}$.

When $r>s:=I(f \diamond x)$, taking a sequence $\left(u_{n}\right) \rightarrow u:=y+s-r$ such that $u_{n} \geq f \diamond x_{n}$ and $\int u_{n}=$ $s_{n}:=r_{n}-r+s$ and setting $y_{n}:=u_{n}+z$, where $z \in L_{1}(S)$ is such that $z \geq 0$ and $I(z)=r-s$, we get $y_{n} \geq u_{n} \geq f \diamond x_{n}$ and $\int y_{n}=\int u_{n}+\int z=r_{n}$, as required.

Then, steps similar to the ones for the proof of Theorem 23 lead to the following result that is an extension to the non locally Lipschitzian case of [8, Thm 2.7.5].

Theorem 26 ([17]) Let $(S, \mathcal{S}, \mu)$ be $\sigma$-finite and let $f: S \times E \rightarrow \mathbb{R}_{\infty}:=\mathbb{R} \cup\{\infty\}$ be a normal integrand finite at $x \in L_{p}(S, E)$ and satisfying condition $(G)$. Let $h: S \times E \rightarrow \overline{\mathbb{R}}$ be the integrand given by $h(s, v):=f_{s}^{C}(x(s), v)$ for $(s, v) \in S \times E$. Then, for all $u \in L_{p}(S, E)$ one has $\left(I_{f}\right)^{C}(x, u) \leq I_{h}(u)$ or

$$
\begin{aligned}
\left(I_{f}\right)^{C}(x, u) & \leq I\left(f^{C} \diamond(x, u)\right), \\
\partial_{C} I_{f}(x) & \subset L_{q}\left(\partial_{C} f \diamond x\right) .
\end{aligned}
$$




\subsection{The case of the directional subdifferential}

For the directional (or Dini-Hadamard or contingent) subdifferential one does not have such simple results. However, under assumption (D) on the integrand, one disposes of an interesting inclusion that can be easily obtained.

Theorem 27 Let $f: S \times E \rightarrow \overline{\mathbb{R}}$ be a normal integrand satisfying condition (D) at $x \in L_{p}(S, E)$ for which $f \diamond x \in L_{1}(S)$. Then, for all $v \in L_{p}(S, E)$ one has

$$
\begin{aligned}
I_{f^{D}}(x, v) & \leq F^{D}(x, v):=\left(I_{f}\right)^{D}(x, v), \\
L_{q}\left(\partial_{D} f \diamond x\right) & \subset \partial_{D} F(x):=\partial_{D} I_{f}(x) .
\end{aligned}
$$

Proof. By assumption (D) there exist $a \in L_{q}(S), b>0$ and a null set $N$ such that

$$
\forall(s, e) \in(S \backslash N) \times E \quad f_{s}(x(s)+e)-f_{s}(x(s)) \geq-a(s)\|e\|-b\|e\|^{p}
$$

with $b=0$ if $p=1$. Given $v \in L_{p}(S, E)$, let $\left(t_{n}\right) \rightarrow 0_{+}$and $\left(v_{n}\right) \rightarrow v$ in $L_{p}(S, E)$ be such that $\left(t_{n}^{-1}[F(x+\right.$ $\left.\left.\left.t_{n} v_{n}\right)-F(x)\right]\right) \rightarrow F^{D}(x, v)$. We set

$$
q_{n}(s):=\frac{1}{t_{n}}\left[f\left(s, x(s)+t_{n} v_{n}(s)\right)-f(s, x(s))\right],
$$

so that $\left(I\left(q_{n}\right)\right) \rightarrow F^{D}(x, v)$. Taking a subsequence $\left(v_{k(n)}\right)$ of $\left(v_{n}\right)$, we may assume that $\left(v_{k(n)}\right) \rightarrow v$ a.e. Let us consider the sequence of nonnegative functions $\left(p_{n}\right)$ defined by

$$
p_{n}(s):=q_{k(n)}(s)+a(s)\left\|v_{k(n)}(s)\right\|+b t_{k(n)}^{p-1}\left\|v_{k(n)}(s)\right\|^{p}
$$

and observe that

$$
\begin{gathered}
\liminf _{n} q_{k(n)}(s)+a(s)\left\|v_{k(n)}(s)\right\|+b t_{k(n)}^{p-1}\left\|v_{k(n)}(s)\right\|^{p} \geq f_{s}^{D}(x(s), v(s))+a(s)\|v(s)\|, \\
\left(\int_{S}\left[a(s)\left\|v_{k(n)}(s)\right\|+b t_{k(n)}^{p-1}\left\|v_{k(n)}(s)\right\|^{p}\right] d \mu(s)\right) \rightarrow \int_{S} a(s)\|v(s)\| d \mu(s)
\end{gathered}
$$

by Hölder's inequality. Applying Fatou's lemma to $\left(p_{n}\right)$ we obtain

$$
I_{f}^{D}(x, v)+I(a\|v\|)=\lim _{n} \int_{S} p_{n}(s) d \mu(s) \geq \int_{S}\left[f_{s}^{D}(x(s), v(s))+a(s)\|v(s)\|\right] d \mu(s),
$$

hence $I_{f}^{D}(x, v) \geq I_{f}(x, v)$. The inclusion $L_{q}\left(\partial_{D} f \diamond x\right) \subset \partial_{D} I_{f}(x)$ ensues.

Let us present a more refined result given in [21]. It uses condition $\left(\mathrm{C}_{p}\right)$ instead of condition (D) and the notion of uniformly integrable sequence. Let us recall it. A sequence $\left(v_{n}\right)$ of $L_{1}(S)$ is said to be uniformly integrable if $\lim _{r \rightarrow \infty} \sup _{n}\left\|1_{\left\{v_{n} \geq r\right\}} v_{n}\right\|_{1}=0$. By [14, Thm 2.29], [21, Lemma 3.2] or [46, Proposition 8.16], this condition implies the following condition and when $\left(v_{n}\right)$ is bounded in $L_{1}(S)$ is equivalent to it:

(E) for every $\varepsilon>0$ there exists some $\delta>0$ such that for all $A \in \mathcal{S}$ satisfying $\mu(A)<\delta$ one has $\int_{A}\left|v_{n}\right| d \mu \leq \varepsilon$ for all $n \in \mathbb{N}$.

The sequence $\left(v_{n}\right)$ is said to be equi-integrable if it satisfies conditions $(\mathrm{E})$ and $\left(\mathrm{E}^{\prime}\right)$, with

(E') for every $\varepsilon>0$ there exists some $T \in \mathcal{S}$ satisfying $\mu(T)<\infty$ such that $\sup _{n}\left\|1_{S \backslash T} v_{n}\right\|<\varepsilon$.

Let us recall that any convergent sequence of $L_{1}(S)$ is equi-integrable and that for any sequence $\left(S_{n}\right)$ in $\mathcal{S}$ and any equi-integrable sequence $\left(v_{n}\right)$ the sequence $\left(1_{S_{n}} v_{n}\right)$ is equi-integrable. We need the following refined Fatou's lemma.

Lemma 28 ([21]) Let $\left(u_{n}\right)$ be a sequence of measurable functions from $S$ to $\overline{\mathbb{R}}$ such that the sequence $\left(v_{n}\right)$ given by $v_{n}=u_{n}^{-}:=\max \left(-u_{n}, 0\right)$ is equi-integrable and bounded in $L_{1}(S)$. Then, if $(S, \mathcal{S}, \mu)$ is $\sigma$-finite, one has

$$
I\left(\liminf _{n} u_{n}\right) \leq \liminf _{n} I\left(u_{n}\right) .
$$


Proof. Let $\left(u_{n}\right)$ be such that $v_{n}=u_{n}^{-}$is equi-integrable and bounded in $L_{1}(S)$. Let us pick some positive integrable function $z$ such that $\int_{S} z d \mu=1$. By [21, Lemma 3.2] the family $\left\{v_{n} / z\right\}$ is uniformly integrable: $\lim _{r \rightarrow \infty} \sup _{n}\left\|1_{\left\{v_{n} \geq r z\right\}} v_{n}\right\|_{1}=0$. Given $\varepsilon>0$ we take $r>0$ such that $\sup _{n}\left\|1_{\left\{v_{n} \geq r z\right\}} v_{n}\right\|_{1} \leq \varepsilon$. Let $w_{n}:=\sup \left(u_{n},-r z\right)$. Observing that $w_{n} \leq 0$ on $S_{n}:=\left\{u_{n} \leq-r z\right\}$ and $w_{n}=u_{n}$ on $S_{n}^{c}:=S \backslash S_{n}$ we have

$$
\begin{gathered}
I\left(w_{n}\right)=I\left(1_{S_{n}} w_{n}\right)+I\left(1_{S_{n}^{c}} w_{n}\right) \leq I\left(1_{S_{n}^{c}} w_{n}\right)=I\left(1_{S_{n}^{c}} u_{n}\right) \\
I\left(w_{n}\right)+I\left(1_{S_{n}} u_{n}\right) \leq I\left(1_{S_{n}^{c}} u_{n}\right)+I\left(1_{S_{n}} u_{n}\right)=I\left(u_{n}\right) .
\end{gathered}
$$

Since $w_{n} \geq-r z$ for all $n$, we can apply Fatou's lemma to $\left(w_{n}\right)$, so that

$$
I\left(\liminf _{n} u_{n}\right) \leq I\left(\liminf _{n} w_{n}\right) \leq \liminf _{n} I\left(w_{n}\right) .
$$

Since $I\left(1_{S_{n}} u_{n}\right)=I\left(-1_{\left\{v_{n} \geq r z\right\}} v_{n}\right)=-I\left(1_{\left\{v_{n} \geq r z\right\}} v_{n}\right)=-\left\|1_{\left\{v_{n} \geq r z\right\}} v_{n}\right\|_{1} \geq-\varepsilon$ we deduce from relation (11) that

$$
\liminf _{n} I\left(w_{n}\right)-\varepsilon \leq \liminf _{n}\left(I\left(w_{n}\right)+I\left(1_{\left\{u_{n} \leq-r z\right\}} u_{n}\right)\right) \leq \liminf _{n} I\left(u_{n}\right) .
$$

Since $\varepsilon$ is arbitrary the result follows.

Theorem 29 Let $f: S \times E \rightarrow \overline{\mathbb{R}}$ be a normal integrand such that the associated integral functional $I_{f}$ on $L_{p}(S, E)$ is calm at $x$ with $f \diamond x \in L_{1}(S)$. Then, the conclusions of the preceding theorem hold.

Proof. Let $\bar{r}>0, c>0$ be such that $I_{f}(x+u)-I_{f}(x) \geq-c\|u\|_{p}$ for all $u \in L_{p}(S, E)$ such that $\|u\|_{p} \leq \bar{r}$. Again, it suffices to prove relation (9) for $v \in L_{p}(S, E)$. Let $\left(t_{n}\right) \rightarrow 0_{+},\left(v_{n}\right) \rightarrow v$ be such that $\lim _{n} t_{n}^{-1}\left(I_{f}\left(x+t_{n} v_{n}\right)-I_{f}(x)\right)=\left(I_{f}\right)^{D}(x, v)$. Taking a subsequence of a given subsequence, we may assume that $\left(v_{n}\right) \rightarrow v$ a.e. and $\left\|t_{n} v_{n}\right\|_{p} \leq \bar{r}$ for all $n \in \mathbb{N}$. Let $\left.q_{n}:=t_{n}^{-1}\left(f \diamond\left(x+t_{n} v_{n}\right)-f \diamond x\right)\right)$ and let $S_{n}:=\left\{s \in S: q_{n}(s) \leq 0\right\}$. The sequence $\left(q_{n}^{-}\right)$is bounded in $L_{1}(S)$ since

$$
\begin{aligned}
\int_{S} q_{n}^{-} d \mu & =-\int_{S} 1_{S_{n}} q_{n} d \mu=\frac{1}{t_{n}} \int_{S}\left(f \diamond x-f \diamond\left(x+t_{n} 1_{S_{n}} v_{n}\right)\right) d \mu \\
& =\frac{1}{t_{n}}\left(I_{f}(x)-I_{f}\left(x+t_{n} 1_{S_{n}} v_{n}\right)\right) \leq c\left\|1_{S_{n}} v_{n}\right\|_{p} \leq c\left\|v_{n}\right\|_{p} .
\end{aligned}
$$

It is equi-integrable since $\left(\left\|v_{n}(\cdot)\right\|^{p}\right)$ is equi-integrable and since for any $A \in \mathcal{S}$ one has

$$
\int_{A} q_{n}^{-} d \mu=\int_{S} \frac{1}{t_{n}}\left(f \diamond x-f \diamond\left(x+t_{n} 1_{A \cap S_{n}} v_{n}\right)\right) d \mu \leq c\left\|1_{A \cap S_{n}} v_{n}\right\|_{p} .
$$

Applying the extended Fatou's lemma we get $I\left(\liminf _{n} q_{n}\right) \leq \liminf _{n} I\left(q_{n}\right)$ and

$$
I\left(f^{D} \diamond(x, v)\right) \leq I\left(\liminf _{n} q_{n}\right) \leq \lim _{n} t_{n}^{-1}\left(I_{f}\left(x+t_{n} v_{n}\right)-I_{f}(x)\right)=\left(I_{f}\right)^{D}(x, v) .
$$

Counter-example. The preceding relations do not hold without some assumption on the integrand, as the following example shows. Let $S:=[0,1]$ with its Lebesgue measure and let the integrand $f: S \times \mathbb{R} \rightarrow \mathbb{R}$ be given by $f(s, e):=e^{3}$. For $n \geq 1$ let $S_{n}:=\left[0, n^{-5}\right]$ and let $v_{n}:=-n^{2} 1_{S_{n}}$ so that $\left\|v_{n}\right\|_{2}=1 / \sqrt{n}$ and $\left(v_{n}\right) \rightarrow 0$. Let $t_{n}=n^{-1 / 3}$. Then $t_{n}^{-1} I_{f}\left(t_{n} v_{n}\right)=-t_{n}^{2} n=-n^{1 / 3}$ and $\left(I_{f}\right)^{D}(x, 0)=-\infty$ for $x:=0$ but since $f^{D}(0, u)=0$ for all $u \in \mathbb{R}$ the conclusion $I_{f^{D}}(x, v) \leq\left(I_{f}\right)^{D}(x, v)$ does not hold.

\section{The case of the limiting subdifferential}

We need the following result. 
Lemma 30 ([1, Thm 8.4.1], [32, Prop. 6.5]) Suppose E is a reflexive Banach space and $\left(M_{n}\right)$ is a sequence of multimaps from $S$ into $E^{*}$ with measurable graphs. Let $M:=\operatorname{seq}-w-\lim \sup _{n} M_{n}$, and let $\overline{\mathrm{co}}(M)$ be the multimap given by $\overline{\mathrm{co}}(M)(s):=\overline{\mathrm{co}}(M(s))$, the weak* closed convex hull of $M(s)$. Then $\overline{\mathrm{co}}(M)$ has a measurable graph and for $q \in[1, \infty[$ one has

$$
\operatorname{seq}-w-\limsup _{n} L_{q}\left(M_{n}\right) \subset L_{q}(\overline{\mathrm{co}}(M)) .
$$

Lemma 31 ([17]) Let $f: S \times E \rightarrow \mathbb{R}_{\infty}$ be a normal integrand satisfying the growth property $(G)$ and let $\left(x_{n}\right)$ be a sequence in $L_{p}(S, E)$ with limit $\bar{x} \in \operatorname{dom} I_{f} \subset L_{p}(S, E)$. If $\left(I_{f}\left(x_{n}\right)\right) \rightarrow I_{f}(\bar{x})$ then $\left(f \diamond x_{n}\right) \rightarrow f \diamond \bar{x}$ in $L_{1}(S, E)$.

Proof. Since $f \diamond \bar{x}$ and $f \diamond x_{n}$ are integrable (for $n$ large enough, so that we may assume that it is the case for all $n$ ) they are finite a.e. Let $g_{n}:=f \diamond \bar{x}-f \diamond x_{n}$, so that, by condition $(\mathrm{G})$, we have $g_{n}(s) \leq \bar{a}(s)+b\left\|x_{n}(s)\right\|^{p}$ a.e., with $\bar{a}(\cdot):=f(\cdot, \bar{x}(\cdot))-a(\cdot) \in L_{1}(S)$ and $b \in \mathbb{R}_{+}$. From any subsequence of $\left(x_{n}\right)$ we can extract a sequence $\left(x_{n}^{\prime}\right)$ that converges to $\bar{x}$ a.e. and such that there exists some $h \in L_{1}(S)$ satisfying $\left\|x_{n}^{\prime}(\cdot)\right\|^{p} \leq h(\cdot)$. Avoiding reindexation, we assume $x_{n}^{\prime}=x_{n}$ and we note that

$$
g_{n}^{+}:=\max \left(g_{n}, 0\right) \leq \bar{a}^{+}+b h .
$$

Since $f_{s}$ is lower semicontinuous on $E$ for a.e. $s$, and since $\left(x_{n}^{\prime}\right) \rightarrow \bar{x}$ a.e., we see that $\left(g_{n}^{+}\right) \rightarrow 0$ a.e. The dominated convergence theorem ensures that $\left(\int_{S} g_{n}^{+}\right) \rightarrow 0$. Now $\left|g_{n}\right|=g_{n}^{+}+g_{n}^{-}$with $g_{n}^{-}:=\max \left(-g_{n}, 0\right)$ and $g_{n}=g_{n}^{+}-g_{n}^{-}$so that

$$
\int_{S} g_{n}^{-}=\int_{S} g_{n}^{+}-\int_{S} g_{n} \rightarrow 0
$$

by the assumption that $\left(I\left(g_{n}\right)\right)=\left(I_{f}(\bar{x})-I_{f}\left(x_{n}\right)\right) \rightarrow 0$. Thus

$$
\left(\int_{S}\left|g_{n}\right|\right)=\left(\int_{S} g_{n}^{+}\right)+\left(\int_{S} g_{n}^{-}\right) \rightarrow 0 .
$$

That shows that $\left(\left\|f \diamond x_{n}-f \diamond \bar{x}\right\|_{1}\right) \rightarrow 0$ since we started from an arbitrary subsequence of $\left(x_{n}\right)$.

In the following statement, given a function $F: X \rightarrow \overline{\mathbb{R}}$ finite at $x \in X$ on a normed space $X$, we denote by $\partial_{L I} F(x)$ the set of weak* limits of sequences $\left(x_{n}^{*}\right)$ such that $x_{n}^{*} \in \partial_{I} F\left(x_{n}\right)$ for some sequence $\left(x_{n}\right) \rightarrow x$ for which $\left(F\left(x_{n}\right)\right) \rightarrow F(x)$.

Proposition 32 Suppose $p \in] 1, \infty\left[, E\right.$ is reflexive and $f: S \times E \rightarrow \mathbb{R}_{\infty}$ is a normal integrand satisfying condition $(G)$. Then, for any $x$ in the domain of $I_{f}$ in $L_{p}(S, E)$ one has

$$
\partial_{L I} I_{f}(x) \subset L_{q}\left(\overline{\mathrm{co}}\left(\partial_{L I} f \diamond x\right)\right) .
$$

Proof. Given $x^{*} \in \partial_{L I} I_{f}(x)$ we pick sequences $\left(x_{n}\right) \rightarrow x,\left(x_{n}^{*}\right) \rightarrow x^{*}$ weakly* with $\left(I_{f}\left(x_{n}\right)\right) \rightarrow I_{f}(x)$, $x_{n}^{*} \in \partial_{I} I_{f}\left(x_{n}\right)$ for all $n \in \mathbb{N}$. Since $x_{n}^{*} \in L_{q}\left(\partial_{I} f \diamond x_{n}\right)$ by 23 , the result is a consequence of the two preceding lemmas, taking $M_{n}:=\partial_{I} f \diamond x_{n}$.

The inclusion $\partial_{F} F \subset \partial_{I} F$ entails the following consequence.

Corollary 33 With the assumptions of the preceding proposition, assume that the integrand $f$ is such that for some null set $N$ of $S$ and all $(s, e) \in(S \backslash N) \times E$ one has $\partial_{I} f_{s}(e)=\partial_{F} f_{s}(e)$, or more generally, that for some $x \in \operatorname{dom} I_{f}$ one has $\overline{\mathrm{co}}\left(\partial_{L I} f \diamond x\right)=\overline{\mathrm{co}}\left(\partial_{L} f \diamond x\right)$. Then one has

$$
\partial_{L} I_{f}(x) \subset L_{q}\left(\overline{\mathrm{co}}\left(\partial_{L} f \diamond x\right)\right)
$$

Note that when $E$ is finite dimensional the additional assumption of the corollary is satisfied whenever $f_{s}$ is D-I regular for a.e. $s$, a rather mild assumption.

On the other hand, one has the following result in which we use the fact that when a Banach space $X$ is separable there is a norm on $X^{*}$ that induces the weak* topology on bounded subsets $([45$, Thm 1.10] for instance). 
Theorem 34 ([24]) Let $f$ be a normal integrand satisfying condition (G). Suppose $E$ is finite dimensional and $L_{p}(S, E)$ is separable. Then, for any $x \in L_{p}(S, E)$ such that $f \diamond x \in L_{1}(S)$ one has

$$
L_{q}\left(\partial_{L} f \diamond x\right) \subset \partial_{L} I_{f}(x) .
$$

If $L_{q}\left(\partial_{L} f \diamond x\right) \neq \varnothing$ then one even has

$$
L_{q}\left(\overline{\mathrm{co}} \partial_{L} f \diamond x\right) \subset \partial_{L} I_{f}(x) .
$$

Combining this theorem with the preceding corollary, we obtain a characterization of $\partial_{L} I_{f}(x)$.

Corollary 35 Let $f$ be a normal integrand satisfying condition $(G)$. Suppose $E$ is finite dimensional and $L_{p}(S, E)$ is separable. If for some $x \in \operatorname{dom} I_{f}$ in $L_{p}(S, E)$ one has $\overline{\operatorname{co}}\left(\partial_{L I} f \diamond x\right)=\overline{\operatorname{co}}\left(\partial_{L} f \diamond x\right)$ and $L_{q}\left(\partial_{L} f \diamond x\right) \neq$ $\varnothing$ then one has

$$
\partial_{L} I_{f}(x)=L_{q}\left(\overline{\operatorname{co}} \partial_{L} f \diamond x\right) .
$$

Corollary 36 Let $f$ be a normal integrand satisfying condition $(G)$. Suppose $E$ is finite dimensional and $L_{p}(S, E)$ is separable. If for some $x \in \operatorname{dom} I_{f}$ in $L_{p}(S, E)$ one has and $L_{q}\left(\overline{\operatorname{co}} \partial_{L} f \diamond x\right)=L_{q}\left(\partial_{C} f \diamond x\right)$, in particular if $f_{s}$ is locally Lipschitzian around $x(s)$ a.e. $s \in S$, then one has

$$
\partial_{L} I_{f}(x)=L_{q}\left(\partial_{C} f \diamond x\right)=\partial_{C} I_{f}(x) .
$$

Proof. Taking into account Theorem 34, that follows from the inclusions $\partial_{L} I_{f}(x) \subset \partial_{C} I_{f}(x) \subset L_{q}\left(\partial_{C} f \diamond x\right)$ (Theorem 26). A slightly different result is given in the next section.

\section{The benefits of regularity}

In the sequel we say that the normal integrand $f$ is $A$-B regular along $x \in L_{p}(S, E)$ if for some null subset $N$ of $S$ one has $\partial_{A} f_{s}(x(s))=\partial_{B} f_{s}(x(s))$ for all $s \in S \backslash N$. We dispose of several results showing that regularity of the integrand $f$ can be transferred to regularity of the integral functional $F:=I_{f}$. The converse is not without interest, but no as important for calculus and we do not consider it. We start with one of the weakest regularity property: D-I regularity or proto-differentiability (or epi-differentiability).

Theorem 37 Suppose the normal integrand $f$ is D-I regular along $x \in \operatorname{dom} I_{f}$ in $L_{p}(S, E)$ and $\partial_{D} F(x) \neq \varnothing$, or, more generally, condition $\left(C_{p}\right)$ holds or $I_{f}$ is calm at $x$. Then $F:=I_{f}$ is $D-I$ regular at $x$ and

$$
\partial_{D} F(x)=L_{q}\left(\partial_{D} f \diamond x\right)=L_{q}\left(\partial_{I} f \diamond x\right)=\partial_{I} F(x) .
$$

If condition $\left(C_{p}\right)$ is satisfied at $x$ and if for $u \in L_{p}(S, E)$ one has $f^{D}(x(\cdot), u(\cdot))=f^{I}(x(\cdot), u(\cdot))$ a.e., in particular if $f^{D}(x(s), \cdot)=f^{I}(x(s), \cdot)$ a.e., then

$$
F^{D}(x, u)=F^{I}(x, u) \quad \forall u \in L_{p}(S, E) .
$$

Proof. Since the inclusion $\partial_{D} F(x) \subset \partial_{I} F(x)$ is always valid, the first assertion stems from the relation $\partial_{D} f \diamond x=\partial_{I} f \diamond x$ and the inclusions $L_{q}\left(\partial_{D} f \diamond x\right) \subset \partial_{D} F(x)$ and $\partial_{I} F(x) \subset L_{q}\left(\partial_{I} f \diamond x\right)$.

In view of the inequality $F^{D}(x, u) \leq F^{I}(x, u)$, the second relation is a consequence of the assumption that $f^{D} \diamond(x, u)=f^{I} \diamond(x, u)$ :

$$
F^{I}(x, u) \leq I\left(f^{I} \diamond(x, u)\right)=I\left(f^{D} \diamond(x, u)\right) \leq F^{D}(x, u)
$$

since $\left(\mathrm{C}_{p}\right)$ or condition (D) implies the last inequality.

Note that under the assumptions of both parts of the theorem, for $\partial=\partial_{D}$ or $\partial=\partial_{I}$ we dispose of representations

$$
\begin{aligned}
\partial F(x) & =L_{q}(\partial f \diamond x), \\
F^{D}(x, u) & =I\left(f^{D} \diamond(x, u)\right)=I\left(f^{I} \diamond(x, u)\right)=F^{I}(x, u) .
\end{aligned}
$$

Let us turn to stronger regularity assumptions. 
Theorem 38 Suppose the normal integrand $f$ is $F-I$ regular along $x \in \operatorname{dom} I_{f}$ in $L_{p}(S, E)$ and condition $\left(D_{x}\right)$ is satisfied. Then $I_{f}$ is $F-I$ regular at $x$ and

$$
\partial_{F} I_{f}(x)=L_{q}\left(\partial_{F} f \diamond x\right)=L_{q}\left(\partial_{I} f \diamond x\right)=\partial_{I} I_{f}(x) .
$$

Proof. Under assumption $\left(D_{x}\right)$, by Theorems 23 and 22 we have

$$
\partial_{I} I_{f}(x) \subset L_{q}\left(\partial_{I} f \diamond x\right) \quad \text { and } \quad L_{q}\left(\partial_{F} f \diamond x\right) \subset \partial_{F} I_{f}(x) .
$$

When $f$ is F-I regular along $x$, i.e. $\partial_{I} f \diamond x=\partial_{F} f \diamond x$, we obtain $\partial_{I} I_{f}(x) \subset \partial_{F} I_{f}(x)$ and since the reverse inclusion is always valid, we get the equalities of the statement.

Theorem 39 Suppose the normal integrand $f$ satisfies conditions $\left(C_{p}\right)$ and $(G)$ and is $C$ - $D$ regular along $x \in \operatorname{dom} I_{f}$. Then, $I_{f}$ is $C$ - $D$ regular at $x$ and

$$
\partial_{C} I_{f}(x)=L_{q}\left(\partial_{C} f \diamond x\right)=L_{q}\left(\partial_{D} f \diamond x\right)=\partial_{D} I_{f}(x) .
$$

If, moreover, $\partial_{C} I_{f}(x)$ is nonempty, for all $u \in L_{p}(S, E)$ one has

$$
\left(I_{f}\right)^{C}(x, u)=I\left(f^{C} \diamond(x, u)\right)=I\left(f^{D} \diamond(x, u)\right)=\left(I_{f}\right)^{D}(x, u) .
$$

Proof. Under our assumptions we have the relation $L_{q}\left(\partial_{C} f \diamond x\right)=L_{q}\left(\partial_{D} f \diamond x\right)$ and the inclusions $L_{q}\left(\partial_{D} f \diamond\right.$ $x) \subset \partial_{D} I_{f}(x)$ and $\partial_{C} I_{f}(x) \subset L_{q}\left(\partial_{C} f \diamond x\right)$. Thus, $\partial_{C} I_{f}(x) \subset \partial_{D} I_{f}(x)$. The inclusion $\partial_{D} I_{f}(x) \subset \partial_{C} I_{f}(x)$ allows us to obtain the first assertion.

To prove the second assertion we note that for a.e. $s \in S$ and $v \in E$ we have

$$
f_{s}^{C}(e, v)=\sup \left\{\left\langle e^{*}, v\right\rangle: e^{*} \in \partial_{C} f_{s}(e)\right\}=\sup \left\{\left\langle e^{*}, v\right\rangle: e^{*} \in \partial_{D} f(e)\right\} \leq f_{s}^{D}(e, v)
$$

for $e:=x(s)$ and equality holds since $f^{D}(x(s), v) \leq f^{C}(x(s), v)$. Thus, for all $u \in L_{p}(S, E)$ we have

$$
I\left(f^{D} \diamond(x, u)\right) \leq\left(I_{f}\right)^{D}(x, u) \leq\left(I_{f}\right)^{C}(x, u) \leq I\left(f^{C} \diamond(x, u)\right)
$$

and $f^{C} \diamond(x, u)=f^{D} \diamond(x, u)$ so that the preceding inequalities are equalities.

Theorem 40 ([24]) Suppose $E$ is finite dimensional, $L_{p}(S, E)$ is separable and the normal integrand $f$ satisfies condition $(G)$ and is $C$ - $L$ regular along $x \in \operatorname{dom} I_{f}$. Then, $I_{f}$ is $C$-L regular at $x$ and

$$
\partial_{C} I_{f}(x)=L_{q}\left(\partial_{C} f \diamond x\right)=L_{q}\left(\partial_{L} f \diamond x\right)=\partial_{L} I_{f}(x) .
$$

Proof. This follows from the inclusions $\partial_{C} I_{f}(x) \subset L_{q}\left(\partial_{C} f \diamond x\right)=L_{q}\left(\partial_{L} f \diamond x\right) \subset \partial_{L} I_{f}(x)$ and $\partial_{L} I_{f}(x) \subset$ $\partial_{C} I_{f}(x)$.

An anonymous referee has kindly observed that the assumption that $E$ is finite dimensional can be replaced with the assumption that $E$ is a separable Banach space.

We arrive at a strong regularity property.

Theorem 41 Suppose the normal integrand $f$ satisfies conditions $(G)$ and $\left(D_{x}\right)$ and is $C$-F regular along $x \in \operatorname{dom} I_{f}$. Then $I_{f}$ is $C$ - $F$ regular at $x$, hence $C$ - D-I-F regular at $x$ and

$$
\partial_{C} I_{f}(x)=L_{q}\left(\partial_{C} f \diamond x\right)=L_{q}\left(\partial_{F} f \diamond x\right)=\partial_{F} I_{f}(x) .
$$

Proof. Under conditions $(\mathrm{G})$ and $\left(D_{x}\right)$, by Theorems 26 and 22 we have

$$
\partial_{C} I_{f}(x) \subset L_{q}\left(\partial_{C} f \diamond x\right) \text { and } L_{q}\left(\partial_{F} f \diamond x\right) \subset \partial_{F} I_{f}(x) .
$$

When $f$ is C-F regular along $x$, i.e. $\partial_{C} f \diamond x=\partial_{F} f \diamond x$, we obtain $\partial_{C} I_{f}(x) \subset \partial_{F} I_{f}(x)$ and since the reverse inclusion is always valid, we get the equalities of the statement. 


\section{$9 \quad$ Legendre functions and integral functionals}

A kind of duality for nonconvex functions has been designed by Ekeland [13], [12] and adapted by the second author to the use of subdifferentials [40], [39], [38]. It encompasses the Fenchel conjugacy. Since the preceding gives some knowledge of the subdifferentials of integral functionals, it is natural to examine its application to such a class of functionals. The case of functionals on $L_{p}(S, E)$ with $p>1$ differs from the case of integral functionals on $L_{1}(S, E)$ considered in [43]. In the latter case, any proper integral functional associated with a normal integrand is an Ekeland function ([43, Thm 18]) in the sense of the following definition.

Definition 42 Given a Banach space $X$, a function $f: X \rightarrow \mathbb{R}_{\infty}:=\mathbb{R} \cup\{\infty\}$ is an Ekeland function with respect to a subdifferential $\partial$, in short a $\partial$-Ekeland function, or just an Ekeland function, if for any $x_{1}, x_{2} \in X$, $x^{*} \in X^{*}$ satisfying $x^{*} \in \partial f\left(x_{1}\right) \cap \partial f\left(x_{2}\right)$ one has $\left\langle x^{*}, x_{1}\right\rangle-f\left(x_{1}\right)=\left\langle x^{*}, x_{2}\right\rangle-f\left(x_{2}\right)$.

Then, the Ekeland transform of $f$ is the function $f^{E}: X^{\prime} \rightarrow \mathbb{R}_{\infty}$ given by $f^{E}\left(x^{*}\right):=\left\langle x^{*}, x\right\rangle-f(x)$ for $x \in(\partial f)^{-1}\left(x^{*}\right)$ when $x^{*} \in \partial f(X), f^{E}\left(x^{*}\right)=+\infty$ for $x^{*} \in X^{\prime} \backslash \partial f(X)$.

Definition 43 The function $f$ is called a (generalized) Legendre function if $f$ is an Ekeland function, if $g:=f^{E}$ is an Ekeland function, if $g^{E}=f$ and if $x^{*} \in \partial f(x) \Leftrightarrow x \in \partial f^{E}\left(x^{*}\right)$.

Any closed proper convex function $f$ is a Legendre function for any subdifferential $\partial$ such that $\partial_{F} f \subset$ $\partial f \subset \partial_{C} f$. Any closed proper concave function $f$ (in the sense that $-f$ is closed proper convex) is a Legendre function for $\partial \in\left\{\partial_{F}, \partial_{D}, \partial_{I}\right\}$ with $f^{E}\left(x^{*}\right)=-(-f)^{*}\left(-x^{*}\right)$; the same is true for $\partial_{C}$ if $f$ is a continuous concave function. Any continuous quadratic function is an Ekeland function and it is a Legendre function when its Hessian is surjective with a direct kernel. Of course, any classical Legendre function in the sense of [46] is a Legendre function.

Moreover, if $A: X \rightarrow Y$ is a surjective continuous linear map between Banach spaces, and if $g: Y \rightarrow \mathbb{R}_{\infty}$ is an Ekeland function, then $f:=g \circ A$ is an Ekeland function and $f^{E}=g^{E} \circ\left(A^{\top}\right)^{-1}$ on $\partial f(X) \subset A^{\top}\left(Y^{*}\right)=$ $(\operatorname{ker} A)^{\perp}$.

Let us examine the application of the preceding concepts to integral functionals. Here we use the coupling between $X:=L_{p}(S, E)$ and $X^{*}:=L_{q}\left(S, E^{*}\right)$ with $\left.p \in\right] 1, \infty\left[, q:=(1-1 / p)^{-1}\right.$. We denote by $f^{E}$ the function $\left(s, e^{*}\right) \mapsto\left(f_{s}\right)^{E}\left(e^{*}\right)$ when $f_{s}$ is an Ekeland function $s$ a.e.

Theorem 44 The integral functional $I_{f}$ associated with an integrand $f$ such that for a.e. $s \in S$ the function $f_{s}$ is an Ekeland function with respect to the incident subdifferential $\partial_{I}$ is an Ekeland function with respect to $\partial_{I}$ and its Ekeland transform is the integral functional associated with the Ekeland transform of the integrand:

$$
\left(I_{f}\right)^{E}\left(x^{*}\right)=I_{f E}\left(x^{*}\right) \quad \forall x^{*} \in L_{q}\left(S, E^{*}\right) .
$$

A similar assertion holds for the circa-subdifferential $\partial_{C}$ provided $f$ satisfies the growth condition $(G)$.

Such a result can be seen as an extension of the pioneering studies of convex integral functionals made by R.T. Rockafellar in [47], [48].

Proof. Given $x^{*} \in L_{q}\left(S, E^{*}\right)$ and $x_{1}, x_{2} \in L_{p}(S, E)$ such that $x^{*} \in \partial_{I} I_{f}\left(x_{1}\right) \cap \partial_{I} I_{f}\left(x_{2}\right)$, we know that $x^{*}(s) \in \partial_{I} f_{s}\left(x_{i}(s)\right)$ a.e. $s \in S$ for $i=1,2$. Then, since $f_{s}$ is an Ekeland function, we have

$$
\left\langle x^{*}(s), x_{1}(s)\right\rangle-f_{s}\left(x_{1}(s)\right)=\left\langle x^{*}(s), x_{2}(s)\right\rangle-f_{s}\left(x_{2}(s)\right) \quad \text { a.e. } s \in S .
$$

Taking the integrals of both sides we see that

$$
\left\langle x^{*}, x_{1}\right\rangle-I_{f}\left(x_{1}\right)=\left\langle x^{*}, x_{2}\right\rangle-I_{f}\left(x_{2}\right) .
$$

This shows that $I_{f}$ is an Ekeland function and that $\left(I_{f}\right)^{E}\left(x^{*}\right)=I_{g}\left(x^{*}\right)$, where $g:=f^{E}$.

Theorem 45 Assume that $E$ is reflexive, that for a.e. $s \in S$ the function $f_{s}$ is a $\partial_{I}$-Legendre function, $g:=f^{E}$ being also a normal integrand, and that $I_{f}$ and $I_{g}$ are calm on their domains. 
(a) If for a.e. $s \in S$ the functions $f_{s}$ and $g_{s}:=f_{s}^{E}$ are $D$-I regular along the elements of $L_{p}(S, E)$ and $L_{q}\left(S, E^{*}\right)$ respectively, then $I_{f}$ is a Legendre function for $\partial_{D}$ and $\partial_{I}$ and is $D-I$ regular.

(b) If $f$ and $g$ are normal integrals satisfying condition $(G)$ for $p$ and $q$ respectively and if (for a.e. $s \in S$ ) $f_{s}$ and $g_{s}$ are corresponding Legendre functions for $\partial_{C}$ that are $D-C$ regular, and such that $I_{f}$ and $I_{g}$ are calm on their domains, then $I_{f}$ is a Legendre function for $\partial_{C}$ and $\partial_{D}$ and is $C-D$ regular.

Proof. (a) Suppose that for a.e. $s \in S$ the function $f_{s}$ is D-I regular along the elements of $L_{p}(S, E)$ and is a Legendre function with respect to $\partial_{I}$. Then, $g_{s}:=f_{s}^{E}:=\left(f_{s}\right)^{E}$ is also a $\partial_{I}$-Ekeland function, and by the preceding, when $g:=f^{E}$ is a normal integrand, $\left(I_{g}\right)^{E}=I_{g^{E}}=I_{f}$ is a $\partial_{I^{-}}$-Ekeland function. Moreover, when $f$ is D-I regular along $x$ and $I_{f}$ is calm on its domain, one has

$$
\begin{aligned}
x & \in \partial_{I} I_{g}\left(x^{*}\right) \Rightarrow x \in L_{p}\left(\partial_{I} g \diamond x^{*}\right) \Rightarrow x^{*} \in L_{q}\left(\partial_{I} f \diamond x\right)=L_{q}\left(\partial_{D} f \diamond x\right) \\
& \Rightarrow x^{*} \in \partial_{D} I_{f}(x) \subset \partial_{I} I_{f}(x)
\end{aligned}
$$

and $\partial_{D} I_{g}\left(x^{*}\right) \subset \partial_{I} I_{g}\left(x^{*}\right)$. Exchanging the roles of $f$ and $g$ have

$$
\begin{aligned}
x^{*} & \in \partial_{D} I_{f}(x) \Rightarrow x^{*} \in \partial_{I} I_{f}(x) \Rightarrow x^{*} \in L_{q}\left(\partial_{I} f \diamond x\right) \Rightarrow x \in L_{p}\left(\partial_{I} g \diamond x^{*}\right)=L_{p}\left(\partial_{D} g \diamond x^{*}\right) \\
& \Rightarrow x \in \partial_{D} I_{g}\left(x^{*}\right) \subset \partial_{I} I_{g}(x),
\end{aligned}
$$

so that these implications are equivalences and $I_{f}$ is a Legendre function for $\partial_{D}$ and $\partial_{I}$.

The proof with the circa-subdifferential is similar.

Remark. In (a), instead of assuming that $f_{s}$ and $g_{s}$ are $D-I$ regular, it suffices to assume that $x^{*} \in$ $L_{q}\left(\partial_{I} f \diamond x\right) \Rightarrow x \in L_{p}\left(\partial_{D} g \diamond x^{*}\right)$ and $x \in L_{p}\left(\partial_{I} g \diamond x^{*}\right) \Rightarrow x^{*} \in L_{q}\left(\partial_{D} f \diamond x\right)$, but such an assumption is not as natural as our assumptions.

Remark. If in (a) one assumes that $f_{s}$ and $g_{s}$ are $F-I$ regular and that for all $x \in L_{p}(S, E)$ (resp. $x^{*} \in L_{q}\left(S, E^{*}\right)$ ) condition $\left(D_{x}\right)$ is satisfied (resp. $\left(D_{x^{*}}\right)$, then $I_{f}$ is a Legendre function for $\partial_{F}$ and is $F-I$ regular.

\section{References}

[1] J.-P. Aubin and H. Frankowska, Set-Valued Analysis, Birkhäuser, Boston, 1990.

[2] J.-M. Bismuth, Intégrales convexes et probabilités, J. Math. Anal. Appl. 42, 639-673 (1973).

[3] M. Bounkhel and L. Thibault, On various notions of regularity of sets in nonsmooth analysis, Nonlin. Anal. 48, 223-246, (2002).

[4] A. Bourass, E. Giner, Kuhn-Tucker conditions and integral functionals, J. Convex Analysis 8 (2), 533-553 (2001).

[5] Ch. Castaing and M. Valadier, Convex Analysis and Measurable Multifunctions. Lecture Notes in Math. vol 580, Springer-Verlag, Berlin and New York (1977).

[6] J.W. Chen and H.C. Lai, Moreau-Rockafellar type theorems for nonconvex non-locally Lipschitz integral functionals on $L_{p}(T, X)$, Optimization 39 (2), 103-122 (1997).

[7] N.H. Chieu, The Fréchet limiting subdifferentials of integral functionals on the space $L_{1}(\Omega, E)$, J. Math. Anal. Appl. 360, (2009), 704-710.

[8] F.H. Clarke, Optimization and Nonsmooth Analysis, Wiley, New York, 1983.

[9] F.H. Clarke, Functional Analysis, Calculus of Variations and Optimal Control, Graduate Texts in Mathematics, 264 Springer, London, 2013.

[10] F.H. Clarke, Yu. S. Ledyaev, R.J. Stern, P.R. Wolenski, Nonsmooth Analysis and Control Theory, Graduate Texts in Mathematics 178. Springer, New York, NY (1998). 
[11] J. Diestel and J.J. Jr Uhl, Vector Measures, Mathematical Surveys 15, American Mathematical Society, Providence, R.I. 1977.

[12] I. Ekeland, Legendre duality in nonconvex optimization and calculus of variations, Siam J. Control Optim 15 (6), 905-934 (1977).

[13] Ekeland I., Nonconvex duality, Bulletin Soc. Math. France, Mémoire n 60 "Analyse non convexe", Pau, 1977, 45-55 (1979).

[14] I. Fonseca and G. Leoni, Modern Methods in the Calculus of Variations: $L_{p}$ Spaces, Springer Monographs in Mathematics, Springer, 2007.

[15] E. Giner, Etudes sur les Fonctionnelles Intégrales, Thesis, Pau, 1985.

[16] E. Giner, Local minimizers of integral functionals are global minimizers, Proc. Amer. Math. Soc. 123 (3), 755-757 (1995).

[17] E. Giner, On the Clarke subdifferential of an integral functional on $L_{p}, 1 \leq p<\infty$, Canadian J. Math. 41 (1), 41-48 (1998).

[18] E. Giner, Michel-Penot subgradients and integral functionals, preprint, Univ. P. Sabatier, Toulouse, April 2006.

[19] E. Giner, Lipschitzian properties of integral functionals on Lebesgue spaces $L_{p}, 1 \leq p<\infty$, Set-Valued Anal. 15, 125-138 (2007).

[20] E. Giner, Subdifferential regularity and characterizations of the Clarke subgradients of integral functionals on Lebesgue spaces $L_{p}, p<+\infty$, J. Nonlinear and Convex Anal., 9 (1), 25-36 (2008).

[21] E. Giner, Calmness properties and contingent subgradients of integral functionals on Lebesgue spaces $L_{p}, 1 \leq p<\infty$, Set-Valued Anal. 17 (3), 223-243 (2009).

[22] E. Giner, Fatou's lemma and lower epi-limits of integral functionals, J. Math. Anal. Appl. 394 (1), 13-29 (2012).

[23] E. Giner, An upper bound for the convergence of integral functionals, J. Convex Anal. 20 (2), 355-376 (2013).

[24] E. Giner, Clarke and limiting subgradients of integral functionals, to appear in J. Convex Anal. (2017).

[25] E. Giner, Some regularity properties of integral functionals, preprint.

[26] E. Giner, A useful underestimate for the convergence of integral functionals, preprint.

[27] F. Hiai and H. Umegaki, Integrals, conditional expectation and martingales of multivalued functions, J. Multivaried Anal. 7 (1977), 149-182.

[28] A.D. Ioffe, Three theorems on subdifferentiation of convex integral functionals, J. Convex Anal. 13 (3-4), (2006), 759-772.

[29] A.D. Ioffe, Theory of subdifferentials, Adv. Nonlinear Anal. 1 (2012), 47-120.

[30] A.D. Ioffe and V.M. Tihomirov, Theory of Extremal Problems, North Holland, Amsterdam (1979).

[31] A. Jourani and L. Thibault, Noncoincidence of the approximate and limiting subdifferentials of integral functionals, SIAM J. Control Optim. 49 (2011), 1435-1453.

[32] M.A. Krasnoselskii, P.P. Zabreiko, E.I. Pustylnik, P.E. Sobolevskii, Integral operators in spaces of summable functions, Noordhoff, Groningen, 1976. 
[33] B.S. Mordukhovich, Variational Analysis and Generalized Differentiation I, Springer-Verlag, Berlin, 2006.

[34] H.V. Ngai and J.-P. Penot, Subdifferentiation of regularized functions, to appear in Set-Valued and Variational Anal. (2016)

[35] J.V. Outrata and W. Römisch, On optimality conditions for some nonsmooth optimization problems over $\mathrm{L}^{p}$ spaces, J. Optim. Th. Appl. 126 (2), 411-438 (2005).

[36] J.-P. Penot, The compatibility with order of some subdifferentials, Positivity 6 (4), (2002), 413-432.

[37] M. Pappalardo, On the image regularity condition, J. Optim. Theory Appl. 121 (2004), no. 3, 673-678.

[38] J.-P. Penot, Generalized Legendre functions and characteristics, preprint, Univ. of Pau (2004). http://lma.univ-pau/data/pub/pub_pdf2005/0529.pdf.

[39] J.-P. Penot, The Legendre transform of correspondences, Pacific J. Optim. 1(1) (2005), 161-177.

[40] J.-P. Penot, Critical duality, J. Global Optim. 40 (nº 1-3) (2008), 319-338.

[41] J.-P. Penot, Softness, sleekness and regularity properties in nonsmooth analysis, Nonlin. Anal. $68 \mathrm{n}^{\circ} 9$, 2750-2768 (2008).

[42] J.-P. Penot, Ekeland duality as a paradigm. in: Advances in applied mathematics and global optimization, 349-376, Adv. Mech. Math., 17, Springer, New York, 2009.

[43] J.-P. Penot, Image space approach and subdifferentials of integral functionals, Optimization 59 (8) (2010), $1-19$.

[44] J.-P. Penot, Towards a new era in subdifferential analysis? in D.H. Bailey et al. (eds), Computational and Analytical Mathematics, Springer Proceedings in Mathematics \& Statistics 50, Springer, New York, (2013) 635-665. DOI 10.1007/978-1-4614-7621-4_29

[45] J.-P. Penot, Calculus Without Derivatives, Graduate Texts in Mathematics 266, Springer, New York (2013).

[46] J.-P. Penot, Analysis: from Concepts to Applications, Universitext, Springer, London, 2016.

[47] R.T. Rockafellar, Integrals which are convex functionals, Pacific J. Maths 24 (1968), 525-540.

[48] R.T. Rockafellar, Integrals which are convex functionals II, Pacific J. Maths 39 (1971), 439-469.

[49] R. T. Rockafellar, Convex integral functionals and duality, in: Contributions to Nonlinear Functional Analysis, edited by E. Zarantonello, Academic Press, New York-London (1971), 215-236.

[50] R.T. Rockafellar, Integral functionals, normal integrands and measurable selections, in Nonlinear Operators and the Calculus of Variations, L. Waelbroeck (ed.), Lecture Notes in Maths 543 (1976), 157-206.

[51] R.T. Rockafellar, Conjugate Duality and Optimization, CBMS-NSF Regional Conferences series in applied mathematics 16, SIAM, Philadelphia, 1974, 1989.

[52] R.T. Rockafellar and R. J.B. Wets, Variational Analysis, Springer, Berlin (1998). 